\title{
Observational evidence for the shrinking of bright maser spots
}

\author{
A. M. S. Richards ${ }^{1}$, M. Elitzur ${ }^{2}$, and J. A. Yates $^{3}$ \\ 1 Jodrell Bank Centre for Astrophysics, School of Physics and Astronomy, Alan Turing Building, University of Manchester, \\ M13 9PL, UK \\ e-mail: amsr@jb.man.ac.uk \\ 2 University of Kentucky, Department of Physics and Astronomy, 600 Rose Street, Lexinton, KY 40506-0055, USA \\ e-mail: moshe@pa.uky.edu \\ 3 Department of Physics and Astronomy, University College London, Gower Street, London, WC1E 6BT, UK \\ e-mail: jyates@star.ucl.ac.uk
}

Received 14 July 2010 / Accepted 18 October 2010

\begin{abstract}
Context. The nature of maser emission means that the apparent angular size of an individual maser spot is determined by the local amplification process as well as by the instrinsic size of the emitting cloud. Highly sensitive radio interferometry images made using MERLIN spatially and spectrally resolve water maser clouds around evolved stars.

Aims. We used measurements of the cloud properties, around the red supergiant S Per and the AGB stars IK Tau, RT Vir, U Her and $\mathrm{U}$ Ori, to test maser beaming theory. In particular, spherical clouds are expected to produce an inverse relationship between maser intensity and apparent size, which would not be seen from very elongated (cylindrical or slab-like) regions.

Methods. We analysed the measured properties of the maser emission in order to estimate the saturation state. We analysed the variation of observed maser spot size with intensity and across the spectral line profiles.

Results. Circumstellar masers emanate from discrete clouds from about one to 20 AU in diameter depending on the star. Most of the maser features have negative excitation temperatures close to zero and modest optical depths, showing that they are mainly unsaturated. Around S Per and (at most epochs) RT Vir and IK Tau, the maser component size shrinks with increasing intensity, although in some cases the slope is shallower than predicted, probably due to shape irregularities and the presence of velocity gradients within clouds. In contrast, the masers around U Ori and U Her tend to increase in size, with a larger scatter.

Conclusions. The water masers from S Per, RT Vir and IK Tau are mainly beamed into spots with an observed angular size much smaller than the emitting clouds. The brighter spots at the line peaks are smaller than those in the wings. This suggests that the masers are amplification-bounded, emanating from clouds which can be approximated as spheres. Many of the masers around U Her and U Ori have apparent sizes which are more similar to the emitting clouds and have less or no dependence on intensity, which suggests that these masers are matter-bounded. This is consistent with an origin in flattened clouds and these two stars have shown other behaviour indicating the presence of shocks which could produce this effect.
\end{abstract}

Key words. masers - stars: AGB and post-AGB - supergiants - circumstellar matter

\section{Introduction}

Maser regions are comprised of many bright, compact spots, each with its own well-defined Doppler velocity. Geometry is an important factor in controlling the brightness of these individual features. Elitzur et al. (1992, hereafter EHM92), suggest that the main difference between the high brightness temperatures of $\mathrm{H}_{2} \mathrm{O}$ masers in star-forming regions and their lower brightness counterparts in evolved stars is the geometry - the pumping scheme and physical conditions are similar in both but the former are characterised by planar shock geometry while the latter are three-dimensional structures that can be modelled as spheres. The spherical maser radius is always limited because loss lines of the pump cycle become optically thick for sufficiently large dimensions, and the maser thermalizes. In contrast, there are no similar limitations on the size of either disk or filamentary masers. In these cases the pumping cycle thermal photons can escape along a short dimension perpendicular to the line of sight while the length along the latter can increase arbitrarily without any effect on the basic inversion efficiency, allowing extremely high brightness temperatures.
Unfortunately, maser geometry cannot be determined directly. As noted in EHM92, from any given direction a spherical maser appears identical to a cylindrical maser aligned along the line of sight. While the length of this equivalent cylinder is the sphere's diameter, its cross section, the spherical maser observed area, is controlled by the maser beaming and determined by the amplification optical depth ("amplification-bounded" maser). The observed area of an amplification-bounded maser varies inversely with the amplification along the line of sight - the stronger is the amplification, the smaller is the observed area. Because the amplification decreases with frequency shift away from line centre, the appearance of a spherical maser varies across the line profile: the observed area is smallest at line centre, increasing toward the line wings (Elitzur 1990). In contrast, the observed area of a cylindrical maser is its actual cross section ("matter-bounded" maser), independent of frequency. Therefore, the maser geometry can be determined indirectly by examining its observed size at different frequencies across the line profile. The cartoon Fig. 1 illustrates these properties. The spherical clouds on the lower left give rise to the amplification-bounded spatial profile shown at the upper left. Some of the clouds shown at lower right are flattened and, if observed edge-on, 

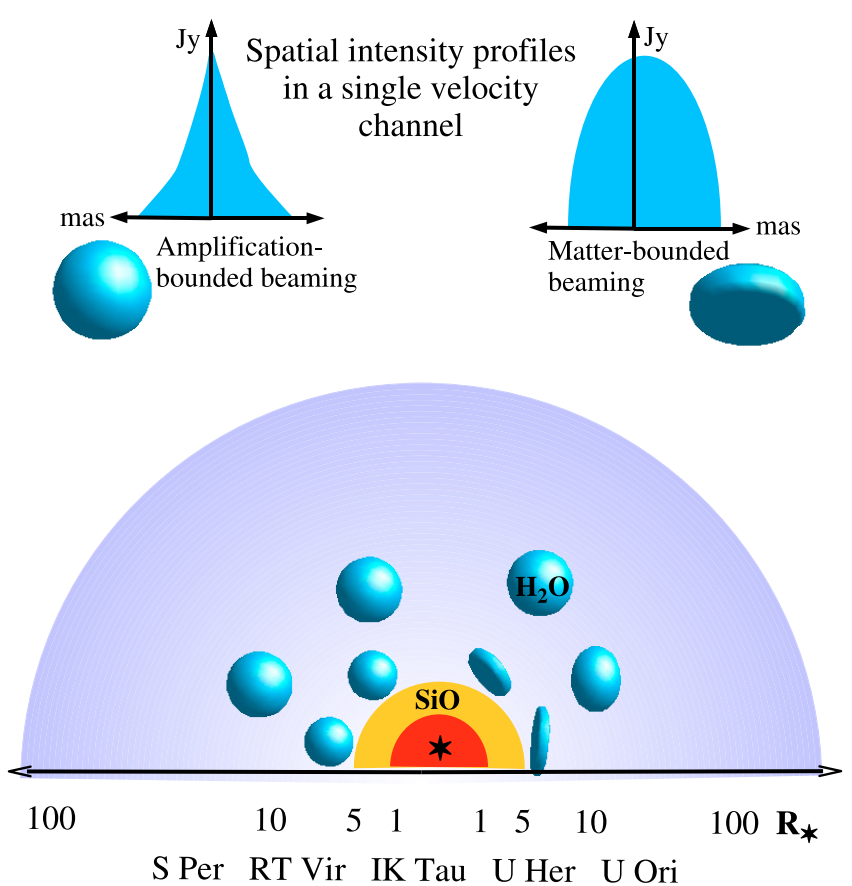

Amplification bounded

Matter bounded

Fig. 1. The lower part of this cartoon shows, on the left, spherical water vapour clouds in a CSE. On the right some clouds are flattened. The upper diagrams show the spatial profiles resulting from maser propagation through a sphere (left) and through a disc seen edge-on (right) The scale in terms of stellar radii is notional. The similarities with the named sources are explained later in this paper.

matter-bounded masing occurs, giving rise to the spectral profile at upper right.

In accordance with the EHM92 proposal, Genzel et al. (1981) report that observed sizes of individual $\mathrm{H}_{2} \mathrm{O}$ maser spots in $\mathrm{W} 51 \mathrm{M}$ are roughly constant across the line profiles, and similar results were obtained for a number of other sources by Walker \& Moran (priv. comm.); the frequency independence of the observed sizes of bright masers in star-forming regions conforms with the matter-bounded geometry expected behind shock fronts.

On the other hand, stellar wind masers are expected to be local regions carved out of the outflow by the requirements of velocity coherence along the line of sight. The resulting geometry is in general fully three-dimensional and amplification-bounded, thus the observed area should increase away from the line centre. Here we test this hypothesis for $\mathrm{H}_{2} \mathrm{O}$ masers in circumstellar envelopes (CSE) of evolved stars.

$\mathrm{H}_{2} \mathrm{O}$ masers at $22 \mathrm{GHz}$ are found around post-mainsequence oxygen-rich asymptotic giant branch (AGB) and red supergiant (RSG) stars, with surface temperatures of 2500$3500 \mathrm{~K}$. AGB stars supporting water masers have radii of $\sim 0.5-3 \mathrm{AU}$ and undergo mass loss at $10^{-6}-10^{-5} M_{\odot} \mathrm{yr}^{-1}$; RSG are around an order of magnitude larger and more prolific. The masers occur within five to a hundred stellar radii $\left(R_{\star}\right)$ of the star. Individual features typically span $1-2 \mathrm{~km} \mathrm{~s}^{-1}$ and the observed size in a single $0.1 \mathrm{~km} \mathrm{~s}^{-1}$ channel is typically a few milli-arcsec (mas).

MERLIN ${ }^{1}$ can produce detailed images of $\mathrm{H}_{2} \mathrm{O}$ maser shells around AGB stars within a few hundred pc, or RSG within a few

\footnotetext{
1 The UK radio interferometer, operated by the University of Manchester on behalf of STFC.
}

Table 1. Properties of the sample stars.

\begin{tabular}{lccccc}
\hline \hline Star & $\begin{array}{c}V_{\star} \\
\left(\mathrm{km} \mathrm{s}^{-1}\right)\end{array}$ & $\begin{array}{c}D \\
(\mathrm{pc})\end{array}$ & $\begin{array}{c}T_{\text {eff }} \\
(\mathrm{K})\end{array}$ & $\begin{array}{c}R_{\star} \\
(\mathrm{mas})\end{array}$ & $(\mathrm{AU})$ \\
\hline S Per & -38.5 & $2312 \pm 35$ & 3550 & $3.5 \pm 1.5$ & 8.0 \\
U Ori & -39.5 & $260 \pm 50$ & 2570 & $5.7 \pm 0.2$ & 1.5 \\
U Her & -14.5 & $266 \pm 22$ & 2630 & $4.8 \pm 0.2$ & 1.3 \\
IK Tau & +34.0 & $250 \pm 20$ & - & $11.2 \pm 1.1$ & 2.8 \\
RT Vir & +18.2 & $135 \pm 15$ & 2924 & $6.2 \pm 0.3$ & 0.8 \\
References & & & & \\
S Per & D87 & M08 & L05 & H94, L05 & \\
U Ori & C91 & C91 & A97 & R06 & \\
U Her & C94 & V07 & A97 & R06 & \\
IK Tau & K87 & 098 & - & M04, R06 & \\
RT Vir & N86 & vL07 & C07 & M04 & \\
\hline
\end{tabular}

Notes. The stellar velocity $V_{\star}$ is given in the Local Standard of Rest (LSR) convention. The distances $D$ used in our calculations are $2.3 \mathrm{kpc}$ for S Per, $266 \mathrm{pc}$ for the Miras, IK Tau, U Ori and U Her and $133 \mathrm{pc}$ for the SRb RT Vir, in order to remain consistent with RYC99 and B+03. The values of $R_{\star}$ for the AGB stars were measured using IR interferometry at $H$ and $K$ bands. The radius of S Per is the average of values deduced from spectral fits.

References. A97: Alvarez \& Mennessier (1997); C91: Chapman et al. (1991); C94: Chapman et al. (1994); C07: Cenarro et al. (2007); D87: Diamond et al. (1987); H74: Humphreys (1974); K87: Kirrane (1987); L05: Levesque et al. (2005); M04: Monnier et al. (2004); M08: Mayne \& Naylor (2008); N86: Nyman et al. (1986); 098: Olofsson et al. (1998); R06: Ragland et al. (2006); V07: Vlemmings \& van Langevelde (2007); vL07: van Leeuwen (2007).

kpc from Earth, without losing significant emission due to missing interferometer spacings. We measured the sizes of individual maser spots by fitting Gaussian components, allowing us to investigate the variations of maser size with intensity, position in the line profiles and other factors. The sample and observational results are described in Sect. 2. We summarise the evidence for maser clumping into discrete clouds and their properties and saturation state in Sect. 3. The variations of the observed size of maser components with intensity and with position in the spectral line profile of clouds are analysed in Sect. 4. This provides diagnostics for the two different maser geometries, explained in Sect. 5. Section 6 summarises compelling evidence that the variations of observed maser sizes across line profiles can be used to distinguish between matter-bounded and amplification-bounded masers.

\section{Observational measurements}

\subsection{Observations}

We have carried out a program of MERLIN observations of 22$\mathrm{GHz} \mathrm{H}_{2} \mathrm{O}$ masers around evolved stars, from 1994 onwards. Four AGB stars (U Her, U Ori, IK Tau and RT Vir) and one RSG (S Per) have good enough visibility plane coverage to resolve the individual maser components. Their relevant properties are summarised in Table 1.

Observational parameters are given in Table 2. The $\sigma_{\text {rms }}$ noise varies greatly not only due to the different durations of observations but to the elevation, the peak (determining the effectiveness of self calibration) and most of all the weather. The 1994 observations and image analysis methods are described in detail by Bains et al. (2003, B+03) for the AGB stars IK Tau, RT Vir, U Her and U Ori and in Richards et al. (1999a, RYC99) for the RSG S Per. Similar procedures were used at later epochs, 
A. M. S. Richards et al.: Observational evidence for the shrinking of bright maser spots

Table 2. Observational measurements of water masers.

\begin{tabular}{|c|c|c|c|c|c|c|c|c|c|c|c|c|c|c|c|}
\hline Star & $\begin{array}{c}\text { Date } \\
\text { (yymmdd) }\end{array}$ & $\begin{array}{l}\text { Dur } \\
(\mathrm{hr})\end{array}$ & $\begin{array}{c}\mathrm{Bm} \\
(\mathrm{mas})\end{array}$ & $\begin{array}{c}\sigma_{\text {rms }} \\
\left(\mathrm{mJyb}^{-1}\right)\end{array}$ & $r_{\mathrm{i}}$ & $\mathrm{U})^{r_{\mathrm{o}}}$ & $\begin{array}{c}v_{\mathrm{i}} \\
(\mathrm{km}\end{array}$ & $\begin{array}{r}v_{\mathrm{o}} \\
\left.\mathrm{s}^{-1}\right) \\
\end{array}$ & $\epsilon$ & $N C$ & $N F$ & $\begin{array}{l}I_{\max } \\
(\mathrm{Jy}) \\
\end{array}$ & $\begin{array}{c}\bar{l} \\
(\mathrm{AU}) \\
\end{array}$ & $N_{\text {fit }}$ & $\begin{array}{c}\overline{\Delta V_{1 / 2}} \\
\left(\mathrm{~km} \mathrm{~s}^{-1}\right)\end{array}$ \\
\hline S Per ${ }^{1}$ & 940324 & 14.5 & 10 & 17 & 55 & 175 & 9.0 & 16 & 0.51 & 1040 & 93 & 72 & $18 \pm 9$ & 50 & $0.77 \pm 0.29$ \\
\hline S Per & 990110 & 13.0 & 10 & 8 & 39 & 175 & 8.0 & 22 & 0.56 & 689 & 100 & 47 & $12 \pm 6$ & 56 & $0.91 \pm 0.34$ \\
\hline U Ori & 940417 & 12.6 & 15 & 12 & 10 & 32 & 2.5 & 7.5 & 0.93 & 64 & 14 & 26 & $2.7 \pm 1.8$ & 2 & $0.65 \pm 0.12$ \\
\hline U Ori & 990109 & 9.1 & 20 & 20 & 12 & 36 & 2.5 & 5.5 & 0.72 & 94 & 13 & 44 & $4.8 \pm 2.6$ & 6 & $0.51 \pm 0.16$ \\
\hline U Ori & 000410 & 8.2 & 18 & 25 & 7 & 36 & 2.0 & 6.0 & 0.65 & 142 & 31 & 8 & $2.7 \pm 2.2$ & 5 & $0.46 \pm 0.23$ \\
\hline U Ori & 010506 & 5.8 & 18 & 27 & 7 & 29 & 2.0 & 5.5 & 0.68 & 165 & 25 & 10 & $4.9 \pm 4.4$ & 10 & $0.74 \pm 0.23$ \\
\hline U Her & 940413 & 13.7 & 15 & 14 & 13 & 47 & 4.0 & 9.5 & 0.69 & 126 & 34 & 22 & $2.3 \pm 1.7$ & 18 & $0.68 \pm 0.35$ \\
\hline U Her & 000519 & 7.6 & 18 & 40 & 10 & 41 & 3.0 & 8.0 & 0.72 & 303 & 44 & 141 & $4.9 \pm 4.4$ & 22 & $0.71 \pm 0.27$ \\
\hline U Her & 010427 & 6.1 & 18 & 35 & 10 & 41 & 3.0 & 8.0 & 0.72 & 212 & 37 & 38 & $3.9 \pm 3.5$ & 16 & $0.62 \pm 0.32$ \\
\hline $\mathrm{IK} \mathrm{Tau}^{1}$ & 940415 & 11.0 & 15 & 10 & 16 & 66 & 5.0 & 16 & 0.82 & 742 & 256 & 25 & $2.0 \pm 0.6$ & 57 & $0.68 \pm 0.28$ \\
\hline IK Tau & 000520 & 7.3 & 15 & 35 & 16 & 72 & 6.0 & 18 & 0.73 & 407 & 72 & 84 & $3.4 \pm 3.1$ & 36 & $0.98 \pm 0.41$ \\
\hline IK Tau & 010427 & 3.9 & 15 & 40 & 16 & 72 & 6.0 & 18 & 0.73 & 175 & 40 & 24 & $3.2 \pm 2.9$ & 19 & $0.81 \pm 0.31$ \\
\hline RT Vir & 940816 & 11.8 & 20 & 12 & 6 & 25 & 4.0 & 10 & 0.65 & 581 & 55 & 394 & $1.4 \pm 0.6$ & 40 & $1.04 \pm 0.38$ \\
\hline RT Vir & 960405 & 4.5 & 12 & 25 & 5 & 19 & 3.5 & 11 & 0.81 & 389 & 58 & 389 & $1.2 \pm 0.9$ & 21 & $0.87 \pm 0.37$ \\
\hline RT Vir & 960421 & 10.8 & 12 & 30 & 5 & 19 & 3.5 & 11 & 0.81 & 400 & 52 & 516 & $1.2 \pm 0.9$ & 20 & $0.90 \pm 0.36$ \\
\hline RT Vir & 960429 & 11.5 & 12 & 15 & 5 & 19 & 3.5 & 11 & 0.81 & 450 & 50 & 526 & $0.9 \pm 0.6$ & 23 & $0.97 \pm 0.41$ \\
\hline RT Vir & 960515 & 9.5 & 12 & 25 & 5 & 19 & 3.5 & 11 & 0.81 & 433 & 41 & 727 & $1.0 \pm 0.8$ & 20 & $1.05 \pm 0.42$ \\
\hline RT Vir & 960524 & 7.7 & 12 & 20 & 5 & 19 & 3.5 & 11 & 0.81 & 401 & 51 & 706 & $0.9 \pm 0.7$ & 19 & $0.88 \pm 0.31$ \\
\hline RT Vir & 960612 & 8.4 & 12 & 35 & 5 & 19 & 3.5 & 11 & 0.81 & 246 & 38 & 791 & $0.9 \pm 0.6$ & 19 & $0.80 \pm 0.25$ \\
\hline
\end{tabular}

Notes. The first 5 columns give the target, date, duration, resolution and sensitivity of each observation. $r_{\mathrm{i}}, r_{\mathrm{o}}, v_{\mathrm{i}}$ and $v_{\mathrm{o}}$ are the inner and outer limits of the $\mathrm{H}_{2} \mathrm{O}$ maser shell. $\epsilon$ is the logarithmic velocity gradient (Sect. 3.1.4). The total number of individual maser components (NC) and features $(N F)$ made up of contiguous series of components are given, along with the maximum total intensity $I_{\max }$ of the brightest spatially distinct component, $\bar{l}$ is the mean size of each feature, $N_{\text {fit }}$ is the number for which a Gaussian spectral profile could be fitted at $>3 \sigma$ and $\overline{\Delta V_{1 / 2}}$ is the mean FWHM of these. All observations used $0.105 \mathrm{~km} \mathrm{~s}^{-1}$ channel width apart from S Per epoch 990110 and IK Tau epochs 000520 and 010427 , when $0.21 \mathrm{~km} \mathrm{~s}^{-1}$ was used. ${ }^{1} N C, N F$ and $v_{\mathrm{o}}$ are probably underestimates since the observational bandwidth was less than the likely velocity extent of emission.

except that phase-reference sources were included after 1999 in order to improve position accuracy. The 1994 observations were made with channels of $0.105 \mathrm{~km} \mathrm{~s}^{-1}$ in a total span of $25 \mathrm{~km} \mathrm{~s}^{-1}$, as was used for all epochs of U Ori, U Her and RT Vir. The spectra of IK Tau and S Per were found to have a larger velocity extent, so double the span was used for these two sources in subsequent epochs, necessitating a doubling of the channel width to $0.21 \mathrm{~km} \mathrm{~s}^{-1}$. The image weightings and restoring beams were adopted to optimise the accuracy of component fitting, which depends mainly on the signal-to-noise ratio (RYC99) as long as the emission has a naturally Gaussian distribution. The masers are well-resolved by MERLIN; the total extent of the 22-GHz emission regions is over a hundred mas, at a resolution of 10-20 mas, and many spectral channels contain multiple distinct patches of maser emission.

RYC99 showed that we detect at least $85 \%$ of the S Per $22-\mathrm{GHz}$ maser emission. Single-dish monitoring with the Pushchino telescope is available within a few months of some epochs for some of our sources. Lekht et al. (2005) detected slightly less flux than we did for S Per in 1994, and more in 1999. Rudnitskij et al. (2000) show that we detected a similar amount of maser emission from U Ori as they did in 1994 and 1999. The single-dish flux of RT Vir within a few months of our observations (Mendoza-Torres et al. 1997; Lekht et al. 1999) was lower than the closest interferometry measurements, but both sets of observations show that it is highly variable, changing flux density by a factor of 2 in a few months.

\subsection{Analysis of maser images}

Detailed results from the 1994 observations are given in RYC99 and $\mathrm{B}+03$ and the large-scale behaviour revealed by more recent observations will be analysed in a future paper. Here, we concentrate on the properties relevant to understanding maser beaming, which are summarised in Table 2.

Identifying individual velocity-coherent maser features in an expanding, turbulent outflow is a difficult task. To this end we developed a two-stage process to disentangle the maser structure properly in velocity and space, first by characterising the spatial distribution of the emission channel by channel and then by examining the spatially-resolved spectral profiles. We start by introducing the first concept, of a maser component: Emission measured in a particular channel centred on velocity $v$, in a contiguous area on the plane of the sky centred on a given direction. The emission pattern of a 3D unsaturated maser in a given frequency interval has the Gaussian shape $\mathrm{e}^{-\left(\theta / \theta_{v}\right)^{2}}$, where $\theta$ is displacement from the direction of the longest chord along the lineof-sight and $\theta_{v}$ is the beaming angle; the observed area is then $A=\pi \theta_{v}^{2}$ (Elitzur 1992). This property of maser emission allows us to obtain accurate measurements by fitting a two-dimensional Gaussian component to each patch of maser emission, in each channel, and deconvolving the restoring beam from the map area as described in RYC99. We selected components brighter than 4 or $5 \sigma_{\text {rms }}$ noise (see RYC99 and $\mathrm{B}+03$ for more details of rejection of artefacts). The resulting fit determines the intensity $I$, position $(x, y)$ and angular FWHM (full width at half maximum) $s_{v}\left(=2 \sqrt{\ln 2} \theta_{v}\right)$ of each maser component.

The uncertainty $\sigma_{s}$ in the component size is the sum in quadrature of the position uncertainties in the $x$ and $y$ directions. There is a lower limit of 0.1 mas to the resolvable component size (Richards 1997) due to dynamic range and calibration limitations. Between $1 \%$ and $40 \%$ of component sizes were upper limits and if $\sigma_{s}>s_{v}$, we took $s_{v}=\sigma_{s}$. Figure 2 shows the distribution of maser component sizes (restricted to $s_{v}>\sigma_{s}$ ) for 


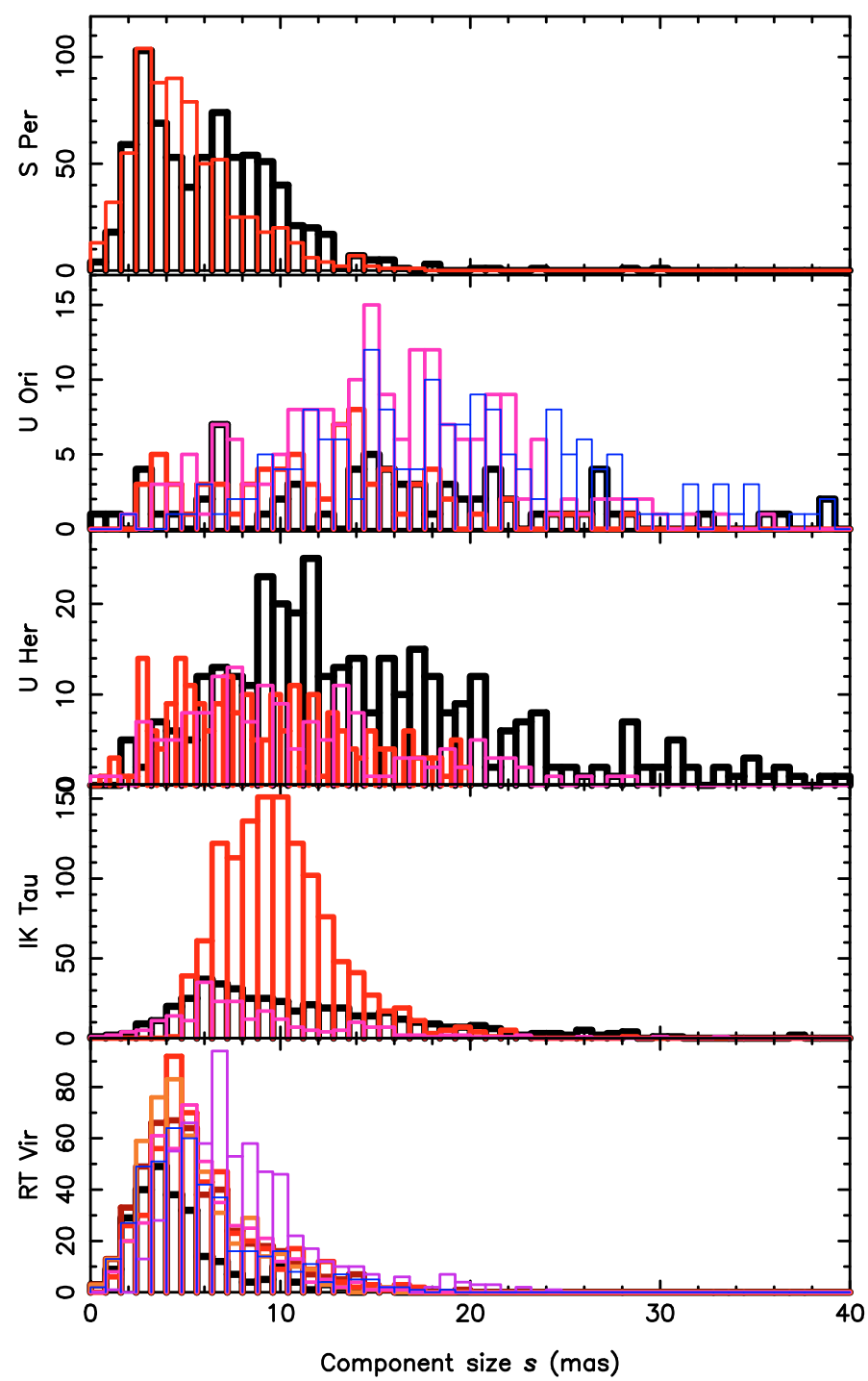

Fig. 2. Histogram of the sizes of $\mathrm{H}_{2} \mathrm{O}$ maser components exceeding the observational error $\left(s_{v}>\sigma_{s}\right)$. The ordinate shows the number of components per size bin. The thickest black line shows the distribution for the epoch with the brightest maximum emission $\left(I_{\max }\right.$, given in Table 2$)$ for each star, with thinner and paler/bluer lines representing epochs with progressively fainter maxima. A few U Her and U Ori components with sizes $>40$ mas are not shown.

each source and epoch. The brightness temperature of a component is

$T_{\mathrm{B}}=\frac{10^{-26} I \lambda^{2}}{2 k_{\mathrm{B}} A}$

where $I$ is in Jy, the wavelength $\lambda=0.013 \mathrm{~m}, k_{\mathrm{B}}$ is Boltzmann's constant and the area $A=(\pi / 4 \ln 2) s_{v}^{2}$ is in steradian.

Figures 3-7 show the variations of $s_{v}$ across the line profiles at each epoch. Figures 8, 12 and 15 illustrate the component distributions in the most well-filled, symmetric CSE (S Per), the most sparse (U Ori) and a well-filled CSE with velocity asymmetry (RT Vir); see plot captions for details. These figures show that maser components lie in series like pearls on a necklace, often curved or twisted.

We introduce the second concept, of a maser feature: a series of adjacent components within a beam width, spanning at least 3 velocity channels. Each feature was defined as possessing a single-peaked spectral profile, unless the emission was too faint to show a clear peak or the profile was truncated by blending. All components were allocated to features consisting of collections of neighbours within a beam width in successive channels. In cases of ambiguity, component allocation was guided by following the smoothest increments of position and flux density from channel to channel. A few, isolated components were rejected as artefacts, being very faint and/or in the position of beam side lobes.

An example feature is enlarged in Fig. 9 (region E in Fig. 8). This shows that the smooth line profile corresponds to a curved position-velocity gradient. The angular FWHM $d$ of the feature is given by the separation of components with intensities closest to half maximum intensity, marked by the short fat red line. The largest angular size $l$ (uncertainty $\sigma_{l}$ ) between its most widely separated components is shown by the long red line in Fig. 9. The interpretation of $d$ is discussed further in Sect. 3.2.4.

We consider the features thus identified as the fundamental building blocks of the maser source. We fitted the spectral profile of each feature, i.e. the component intensity $I(v)$ as a function of $v$, with a Gaussian curve. This is an approximation to the line shape of unsaturated masers, adequate to half maximum. This fitting yields the line FWHM $\Delta V_{1 / 2}$, and the corresponding uncertainty $\sigma_{\Delta V_{1 / 2}}$, as well as the central velocity, the peak model intensity and their uncertainties. The relevant measurements are given in Table 2, along with the number of features at each epoch with fits better than $3_{\sigma}$.

We show additional enlargements of features in Figs. 10, 11, 13, 14, 16 and 17 as clear (but otherwise unexceptional) illustrations of different relationships between $s_{v}$ and intensity or position in the line profile, discussed in Sect. 4. Figures 10 and 11 show multiple spectral peaks in spatially adjacent features which probably emanate from a single physical region, discussed in Sect. 3.1.1.

In summary, the individual maser components represent separate patches of emission as sampled in the observed velocity channels. The components are found to be clustered in position and velocity, often forming smooth Gaussian spectral profiles, termed features. Individual features thus defined are the objects to be compared with maser theory. Table 2 gives the number of components and features in each source.

\section{Properties of maser clouds}

\subsection{Dense clumps in the stellar winds}

The evidence for the origins of $22-\mathrm{GHz}_{2} \mathrm{O}$ maser emission in discrete, density-bounded clouds, as part of a fragmented, expanding wind, is summarised in this section. RYC99 and B+03 explained in more detail why we think that each feature (or occasionally a series of a few features, in the RSG) corresponds to a physically discrete, density bounded cloud, not solely a "spooky" accident of the velocity field or turbulence (Strelnitski 2007).

\subsubsection{Maser components form distinct features with Gaussian spectral profiles}

The relationship between component positions and velocities is illustrated for S Per (Figs. 8 and 10), U Ori (Figs. 12 and 13) and RT Vir (Figs. 15 and 16). Many features show an orderly (but not necessarily linear) distribution of components, with an internal gradient of velocity with position. These gradients do not show any large-scale alignment. The average line widths $\Delta V_{1 / 2}$ for the feature spectral profiles for each star are given in Table 2 . The 
A. M. S. Richards et al.: Observational evidence for the shrinking of bright maser spots

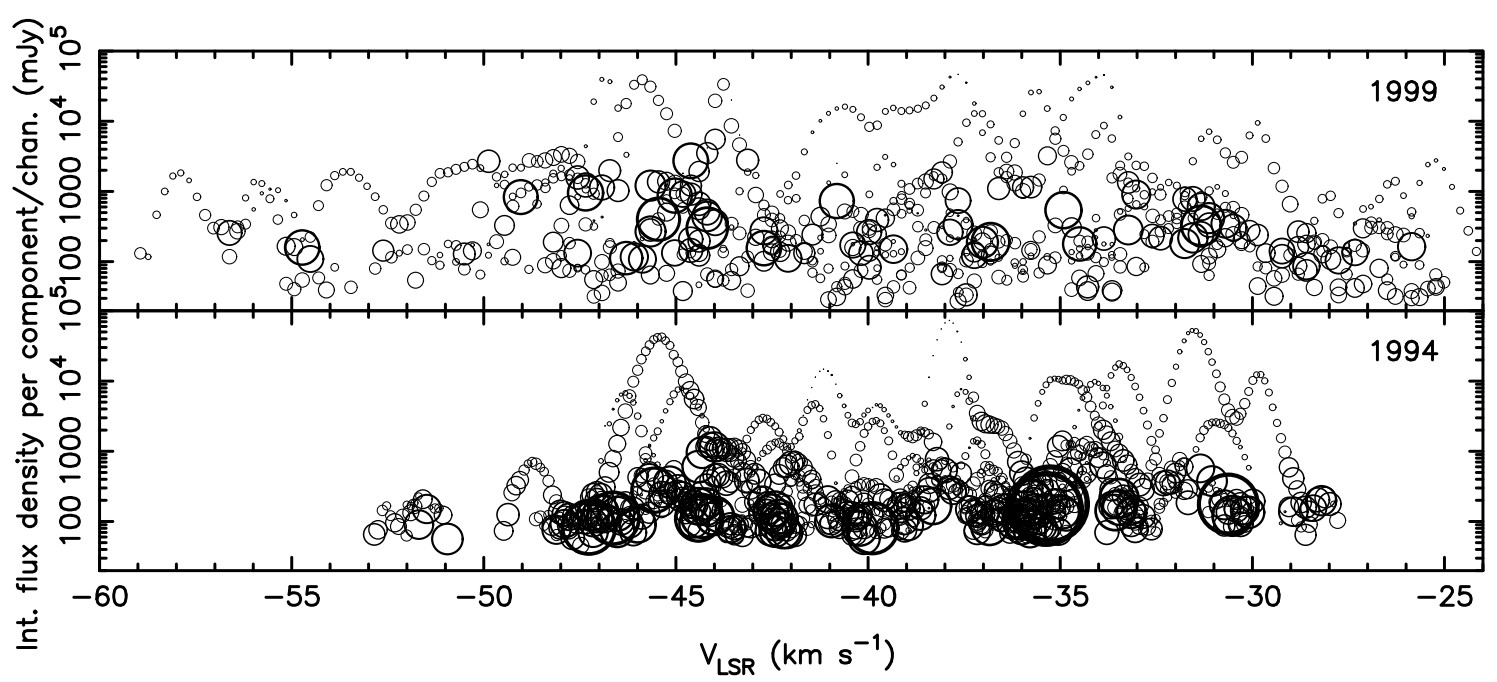

Fig. 3. The components making up the velocity profiles of $\mathrm{H}_{2} \mathrm{O}$ maser features of $\mathrm{S}$ Per in 1999 and 1994 . The symbol diameter is proportional to the observed angular FWHM $s_{v}$ of the components.

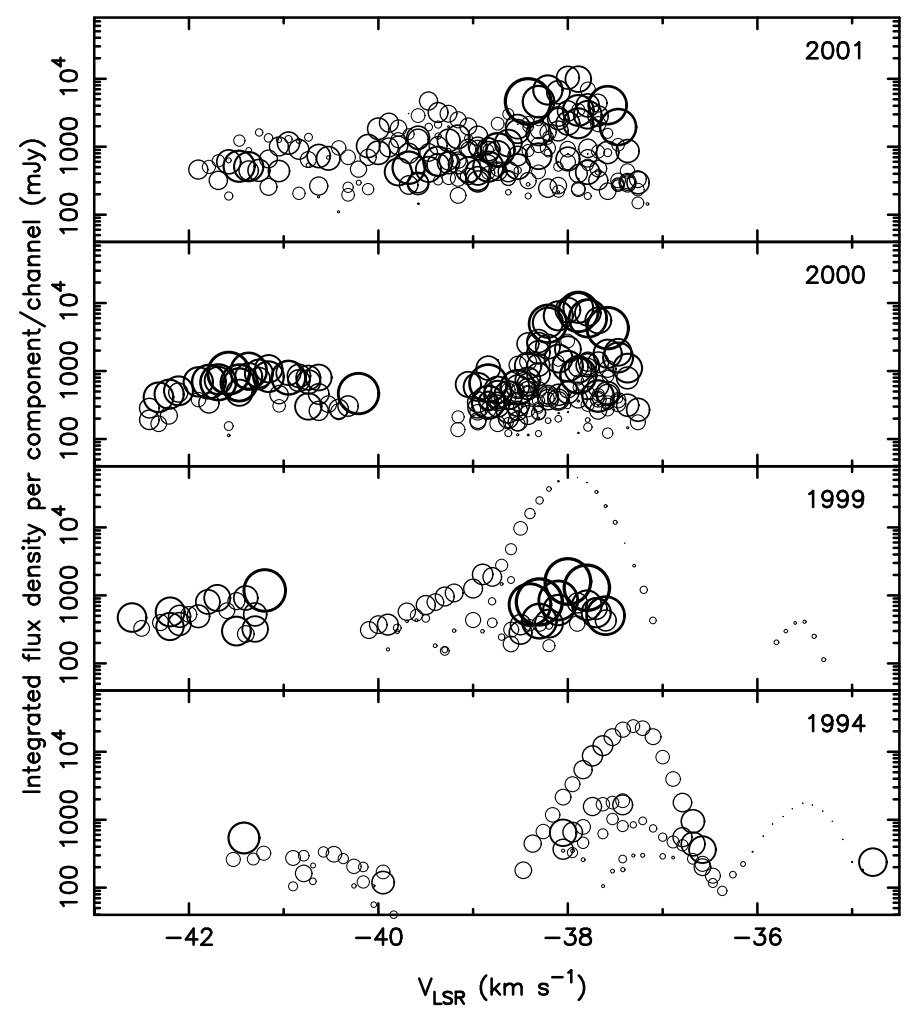

Fig. 4. The velocity profile of U Ori, see Fig. 3 for details.

gas temperature $T$ in the CSE of AGB stars is likely to drop from around $1000 \mathrm{~K}$ at $r_{\mathrm{i}}$ to $400 \mathrm{~K}$ at $r_{\mathrm{o}}$, depending on the thickness of the shell (Zubko \& Elitzur 2000).

The temperature at distance $r$ from the star can be approximated as $T \propto r^{-0.4}$, so the thermal Doppler line width $\Delta V_{\mathrm{D}} \propto$ $\sqrt{T} \propto r^{-0.2} . \Delta V_{\mathrm{D}}$ lies in the range 1.6-1.2 $\mathrm{km} \mathrm{s}^{-1}$ between $r_{\mathrm{i}}$ and $r_{\mathrm{O}}$. The weak temperature dependence means that even if RSGs and their CSEs are slightly hotter than AGB stars (Table 1), this has little effect on maser amplification paths. $\Delta V_{\mathrm{D}}$ exceeds $\Delta V_{1 / 2}$, so maser amplification is likely to take place through the entire depth of each feature.

Features around AGB stars are almost always distinct in position or velocity (or both) and we conclude that they correspond

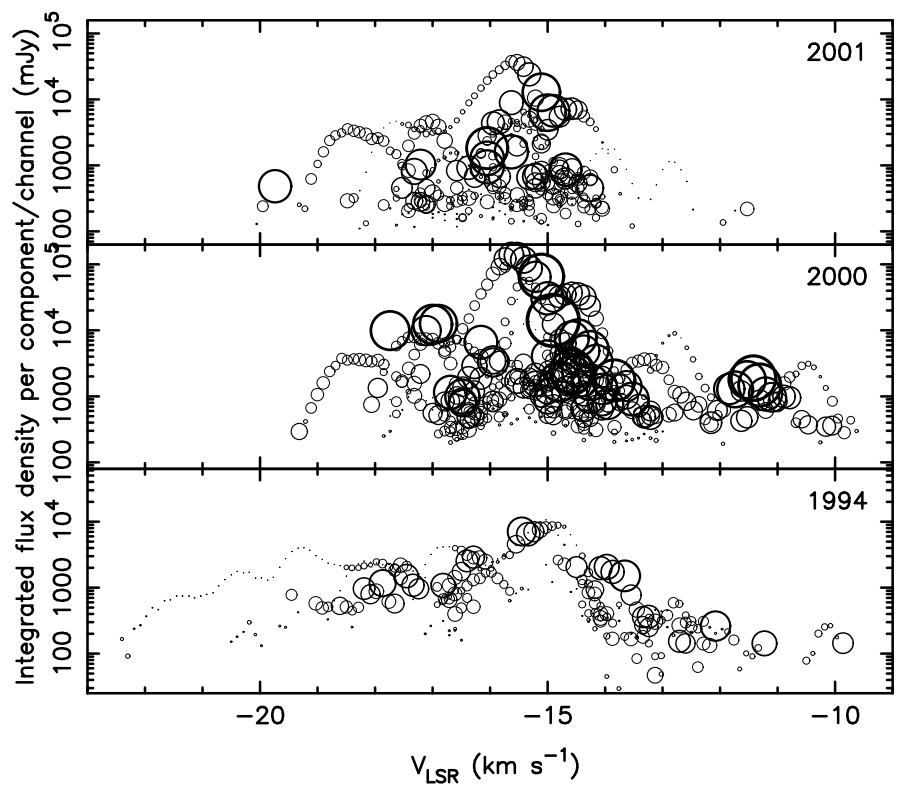

Fig. 5. The velocity profile of U Her, see Fig. 3 for details.

to separate clouds. A few features around RSGs form groups such as the example in Fig. 8 region A. This appears to be a single cloud containing 5-6 multiple, adjacent spectral peaks (Fig. 11), i.e. multiple features in our terminology. These large clouds have a velocity span $>\Delta V_{\mathrm{D}}$ and RYC99 showed that their velocity gradients, rather than their total depths, probably limits their maser amplification. The majority (80-90\%) of features around RSGs appear to emanate from separate clouds.

\subsubsection{The angular extent of features gives the clouds physical sizes}

Emission from successive velocity channels sampling a quiescent sphere would be centred on a common point on the sky. Alternatively, a long thin line of observed maser components would be produced by a spherical cloud with a linear internal velocity gradient. The patterns we observe could be produced by spherical clouds with curved velocity gradients. It seems likely 


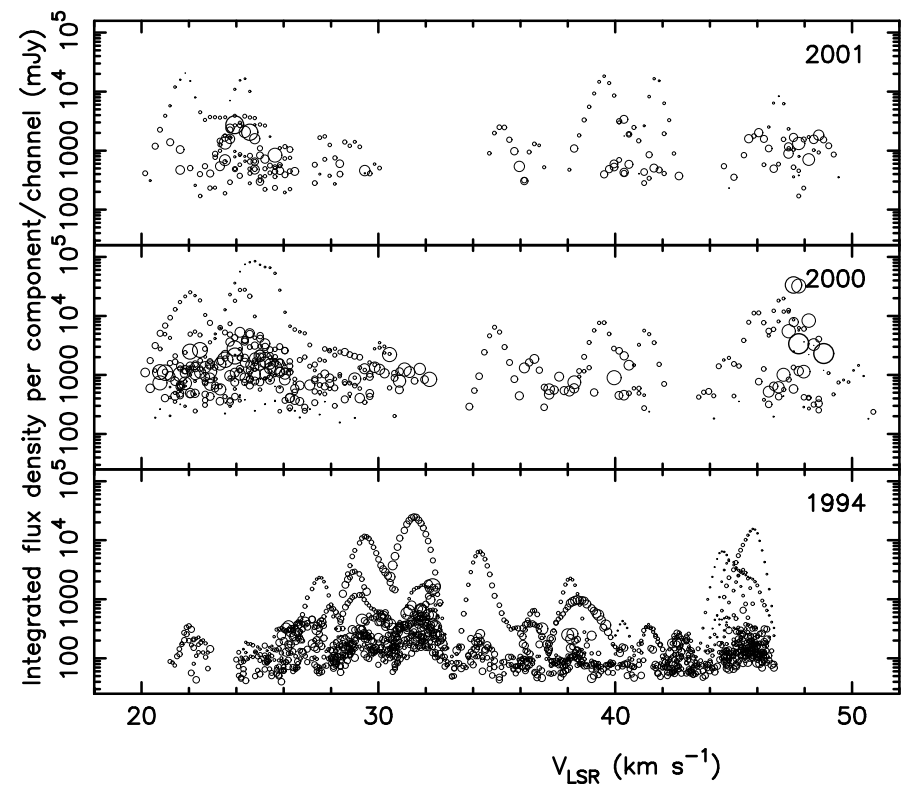

Fig. 6. The velocity profile of IK Tau, see Fig. 3 for details.

that MERLIN is detecting all the maser emission (Sect. 2.1) so $l$ is close to the full extent of the maser features with significant position-velocity gradients. In other cases, $l$ is a lower limit, but individual clouds cannot exceed about $20 \bar{l}$ in any direction or they would not fit inside the observed maser shell inner and outer radii.

The clouds are not necessarily spherical but could be amoeboid or have any other shape. Unfortunately we cannot measure all three dimensions of each cloud directly so we use $l$ (converted to units of length at the appropriate distance for each star) as the physical diameter of a feature in any direction, indicating the most likely biases this causes where relevant. The analysis in this paper (e.g. Sect. 5) will provide further clues to the shapes of clouds.

\subsubsection{Maser clouds persist for many months or years}

The distribution of components within some features forms distinctive patterns which can be recognised (with some distortion) at multiple epochs. Close similarities can be seen in some instances, such as the RT Vir feature shown in Fig. 16 (one of 11 features matched at all 6 epochs of observation taken over 10 weeks). The S Per features seen in Fig. 8 region A, also shown in Fig. 10, appear to have rotated about $60^{\circ}$ anticlockwise during 5 years. The epochs were aligned using the centres of expansion (found as described in RYC99), combined, relative uncertainties $\sim 6.5$ mas. Proper motion studies of the AGB stars show that masing from their $\mathrm{H}_{2} \mathrm{O}$ clouds can be tracked for between a few months and a couple of years (Richards et al. 1999b; Yates \& Cohen 1994). The larger RSG clouds can be tracked for 5 to 10 or more years (Richards et al. 1996; Richards et al. 1998; Murakawa et al. 2003).

\subsubsection{Water maser clouds are much denser than their surroundings}

The $\mathrm{H}_{2} \mathrm{O}$ masers propagate in approximately spherical shells with inner and outer radii of $r_{\mathrm{i}}$ and $r_{\mathrm{o}}$, across which the expansion velocity increases from $v_{\mathrm{i}}$ to $v_{\mathrm{o}}$. Multi-epoch and proper motion measurements suggest that the $\mathrm{H}_{2} \mathrm{O}$ maser shells are

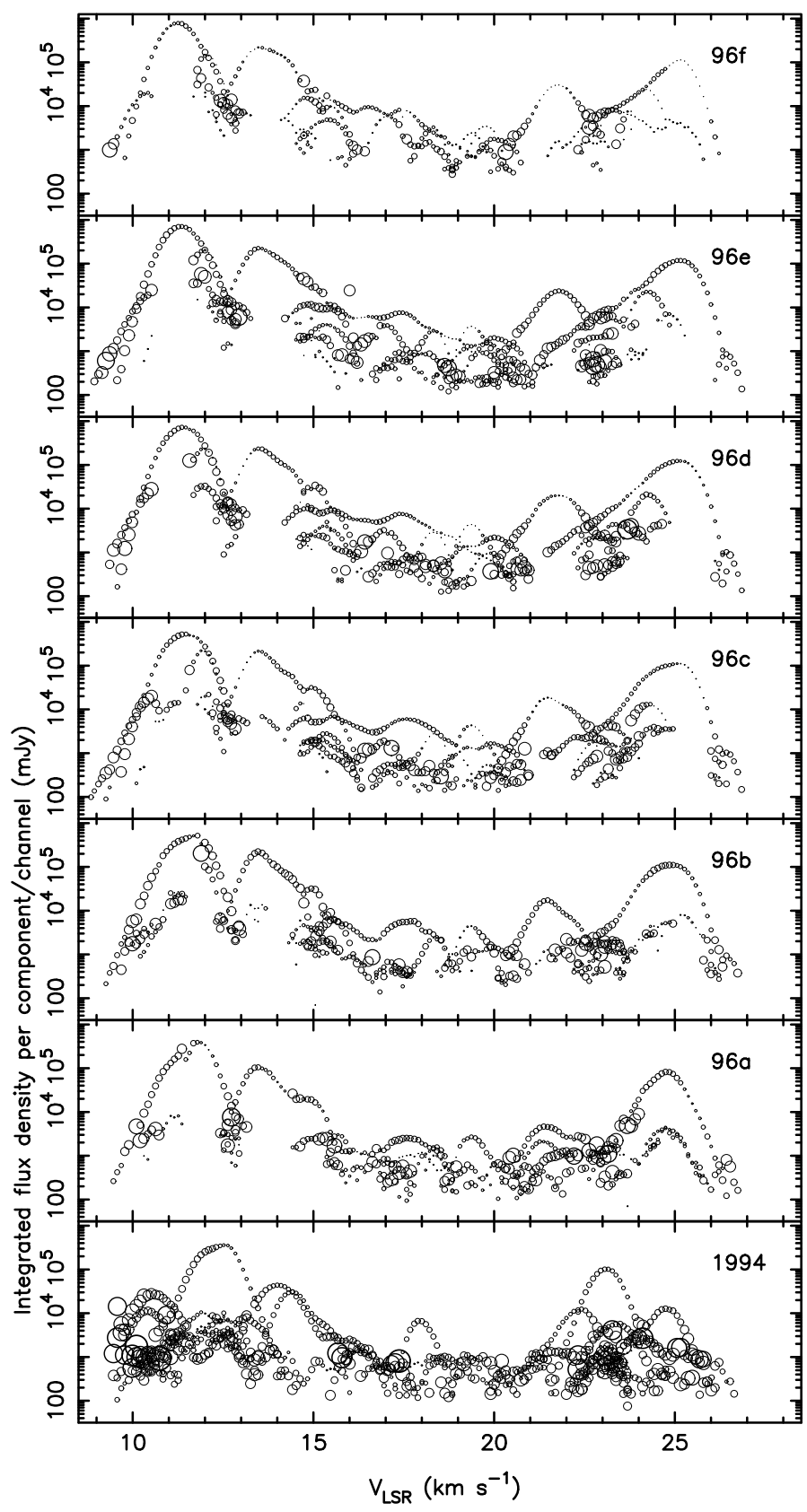

Fig. 7. The velocity profile of RT Vir, see Fig. 3 for details. 96a to $f$ indicate the six successive epochs of observation in 1996.

approximately spherically symmetric (Murakawa et al. 2003; Richards et al. 1999b). This allowed Murakawa et al. (2003, their Eq. (1)) to solve the three-dimensional structure of the circumstellar envelopes and find the distance of each cloud from the star, $r$.

The wind acceleration through the shell is parametrised by the logarithmic velocity gradient $\epsilon=\log \left(v_{\mathrm{o}} / v_{\mathrm{i}}\right) / \log \left(r_{\mathrm{o}} / r_{\mathrm{i}}\right)$. Alcock \& Ross (1986) proposed that if initially spherical, dusty clouds are driven away from a star by radiation pressure on grains, at a constant velocity, their diameter in the radial, outflow direction will be unchanged but their diameter in the tangential direction will increase in proportion to distance from the star. If the wind is accelerating, then the tangential and radial axes (with respect to the star) of a cloud expanding under radiation 


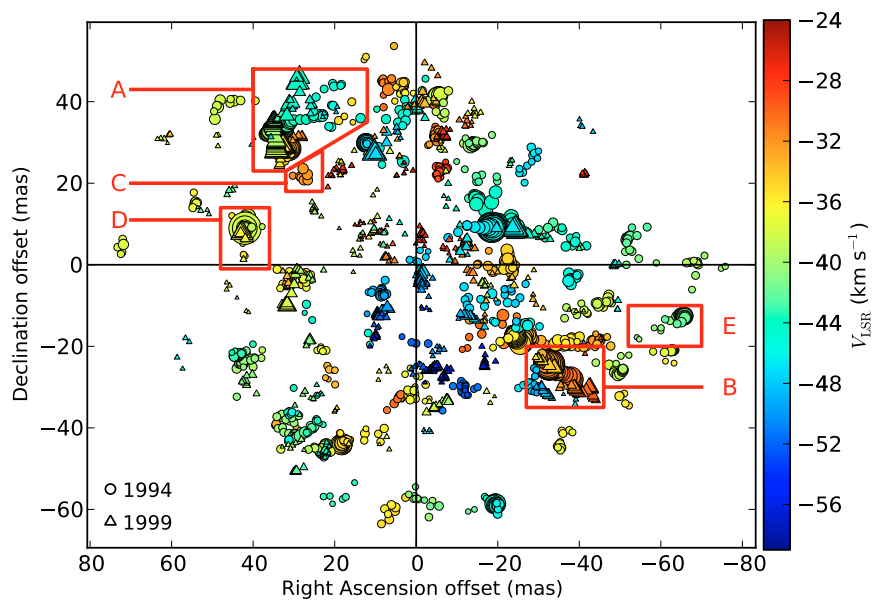

Fig. 8. Each symbol represents an $\mathrm{H}_{2} \mathrm{O}$ maser component in a single channel observed towards S Per in $1994(\circ)$ or $1999(\triangle)$. The diameter is proportional to the square root of total intensity. The colour is proportional to the velocity as shown. The red boxes enclose features which are shown in more detail in Figs. 9-11.
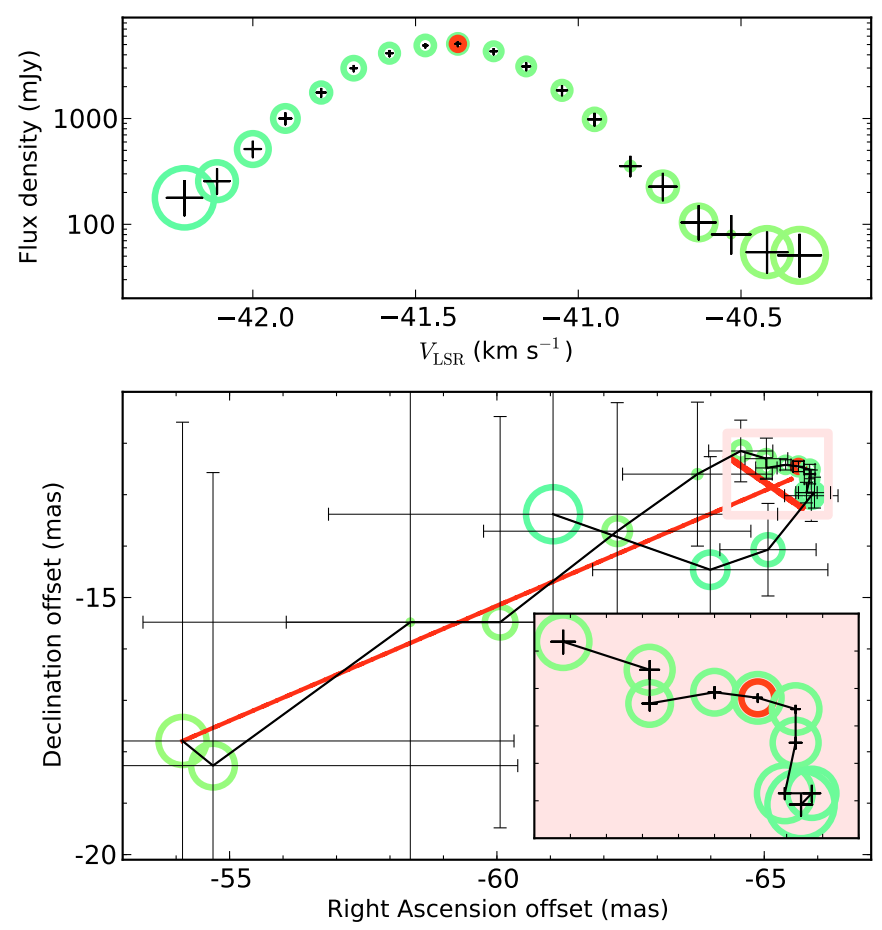

Fig. 9. Properties of the maser feature E in Fig. 8, with the same coloring scheme. Top: feature line profile. The symbol diameters are proportional to $s_{v}$ (two are very small); the error bars show the size uncertainty $\sigma_{s}$. Bottom: detailed map of the components comprising the feature. The diameter of each symbol is $10 \%$ of $s_{v}$ and the error bars represent the position offset uncertainties. The thin black line joins the components in velocity order. The long thin red line denotes the largest angular size $l$ of the feature. The short fat red line shows the separation of the components with flux densities closest to the half-maximum of the feature, $d$. The expanded-scale inset (total size $1.7 \times 1.2$ mas, ticks at 0.2 mas intervals) shows the area in the pink box, which contains the components brighter than half maximum, with component size error bars. The brightest component is outlined in red, also marked on the top plot.

pressure will grow as $r / r_{\mathrm{i}}$ and $\left(r / r_{\mathrm{i}}\right)^{\epsilon}$, respectively. If the clouds are spherical at $r_{\mathrm{i}}$, their tangential/radial aspect ratio will evolve as $\left(r / r_{\mathrm{i}}\right)^{1-\epsilon}$. The cloud number density, relative to that at the inner radius, is given by $n(r)=n_{r_{\mathrm{i}}} \times\left(r / r_{\mathrm{i}}\right)^{-(2+\epsilon)}$.
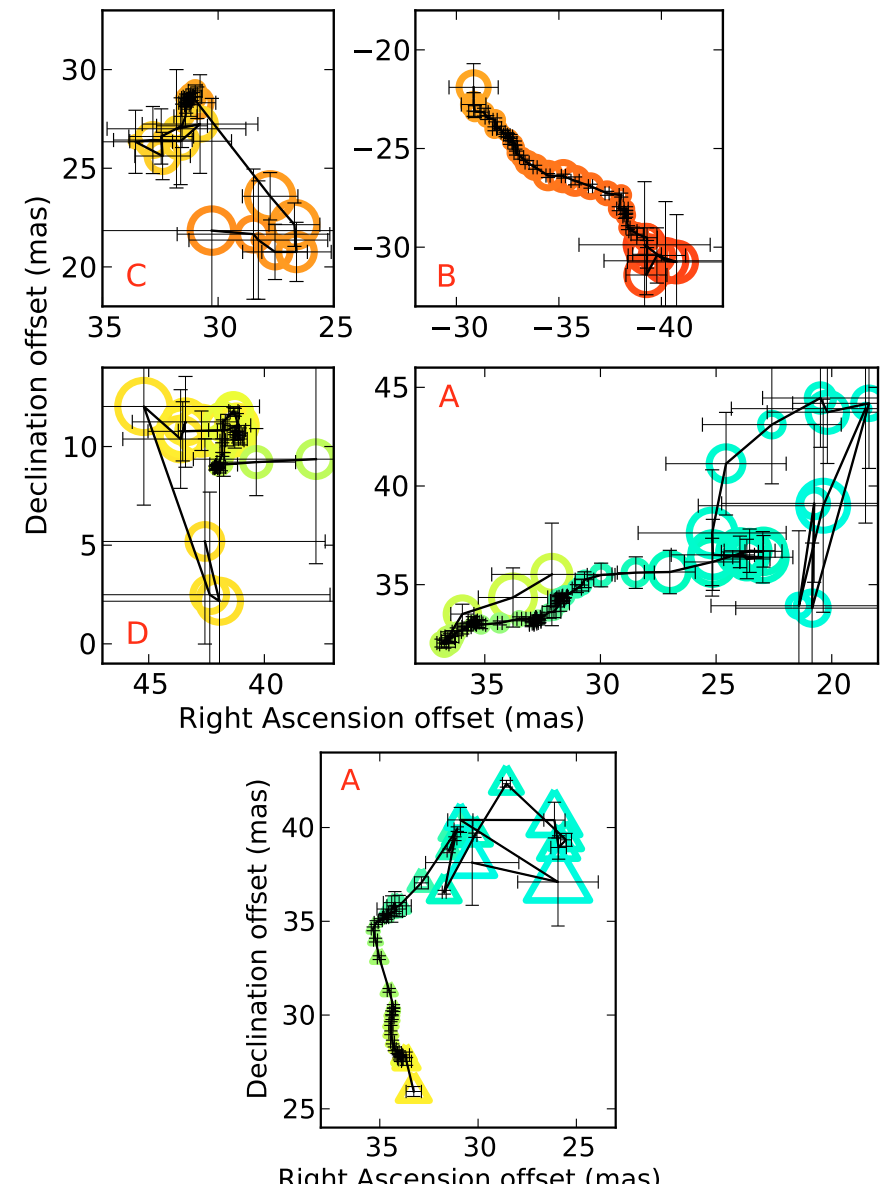

Fig. 10. The angular size, distribution and position uncertainties of the maser components making up the boxed features A-D around S Per in Fig. 8, as measured in 1994 (upper and middle plots, circles) and the features in A as measured in 1999 (lower plot, triangles). The same velocity colour scheme is used. Region A contains 6 features in 1994 and 5 in 1999; the other regions contain 2 features, as can be seen from the spectral peaks in Fig. 11. The diameter of each symbol is $10 \%$ of $s_{v}$. The black lines join the components in velocity order. The position uncertainties are shown.

The inner radius $r_{\mathrm{i}}$ is determined by the distance from the star where the wind density falls below the collisional quenching density for the 22- $\mathrm{GHz} \mathrm{H}_{2} \mathrm{O}$ maser (Cooke \& Elitzur 1985; Cohen 1987), at a number density of $n\left(r_{\mathrm{i}}\right) \approx 5 \times 10^{15} \mathrm{~m}^{-3}$ $\left(\approx 5 \times 10^{9} \mathrm{~cm}^{-3}\right)$. The mass loss rate $\dot{M}$ which would be needed to produce $n\left(r_{\mathrm{i}}\right)$ is one or two orders of magnitude greater than the mass loss rates measured from IR and $\mathrm{CO}$ observations (see references in RYC99 and B+03).

This apparent contradiction is overcome by considering the volume filling factors of the $\mathrm{H}_{2} \mathrm{O}$ maser clouds. The number of clouds $N C$ and their sizes $l$ are given in Table 2 and the filling factors are given by $N C \times l^{3} /\left(r_{\mathrm{o}}^{3}-r_{\mathrm{i}}^{3}\right)$. S Per has the highest factor of $1.5 \%$ (RYC99), RT Vir and IK Tau have factors of about $0.4 \%$ and $U$ Ori and $U$ Her have factors of about $0.1 \%(B+03)$. This leads to realistic values of $\dot{M}$ if the clouds are surrounded by gas at $\sim 1 / 50$ of the cloud number density. This implies that the actual average feature volume per CSE must be no more than a factor of about two greater than that based on the average measured $\bar{l}$, or the mass in features would exceed the total mass loss rate. 

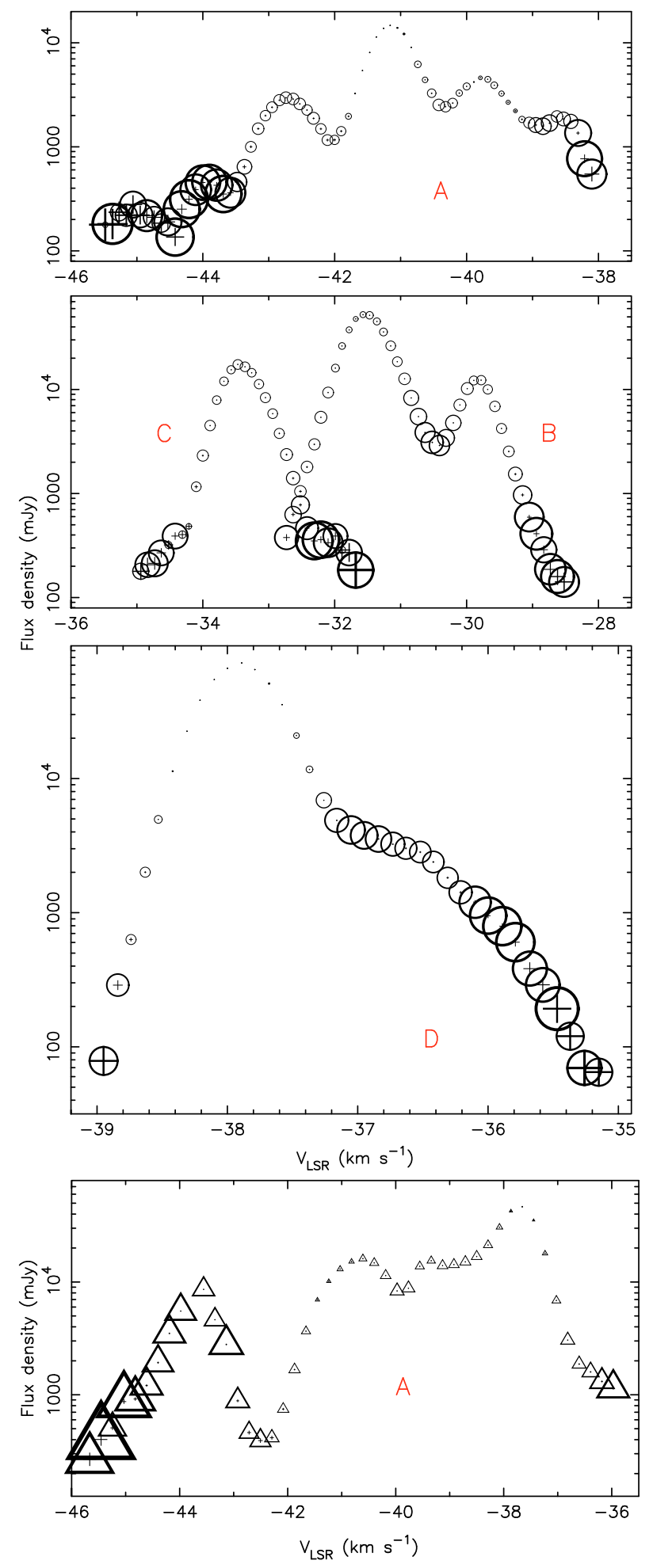

Fig. 11. Top to bottom: the line profiles of features seen around S Per in 1994 (circles) in the regions labelled A, B, C and D in Fig. 8, and in 1999 (triangles) in the region labelled A in Fig. 8. The diameters of the circles/triangles and of the crosses are proportional to the observed angular FWHM $s_{v}$ of the maser components and to $\sigma_{s}$, respectively.

\subsection{Maser optical depth and saturation state}

In this Section, we use our measurements to provide estimates of the parameters determining the maser optical depths, which leads us to infer that the masers are mostly unsaturated. We provide estimates of the uncertainties in our measurements and the

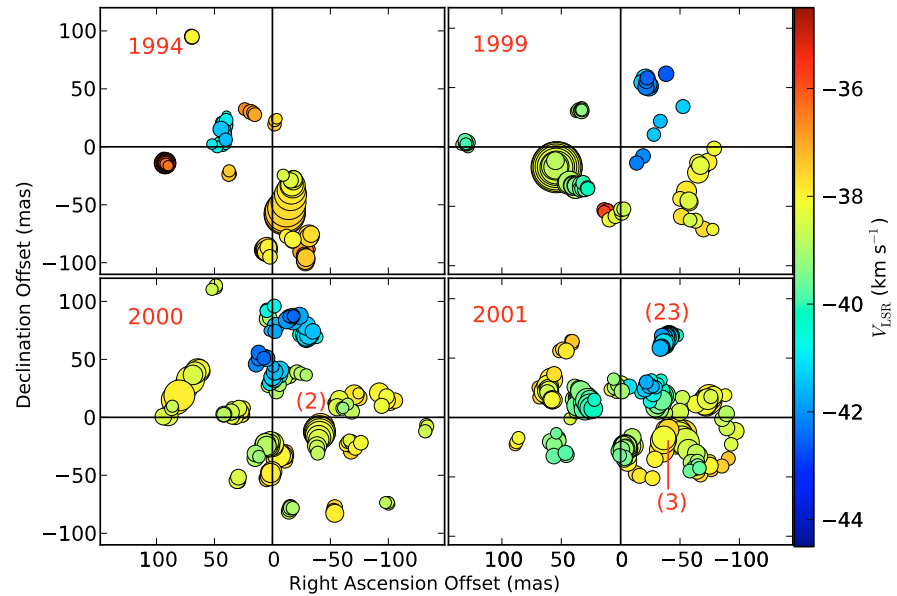

Fig. 12. Each symbol represents an $\mathrm{H}_{2} \mathrm{O}$ maser component in a single channel observed towards U Ori, at the 4 epochs indicated. The diameter is proportional to the square root of total intensity. The colour is proportional to the velocity as shown. The numbered features are shown in more detail in Figs. 13 and 14.

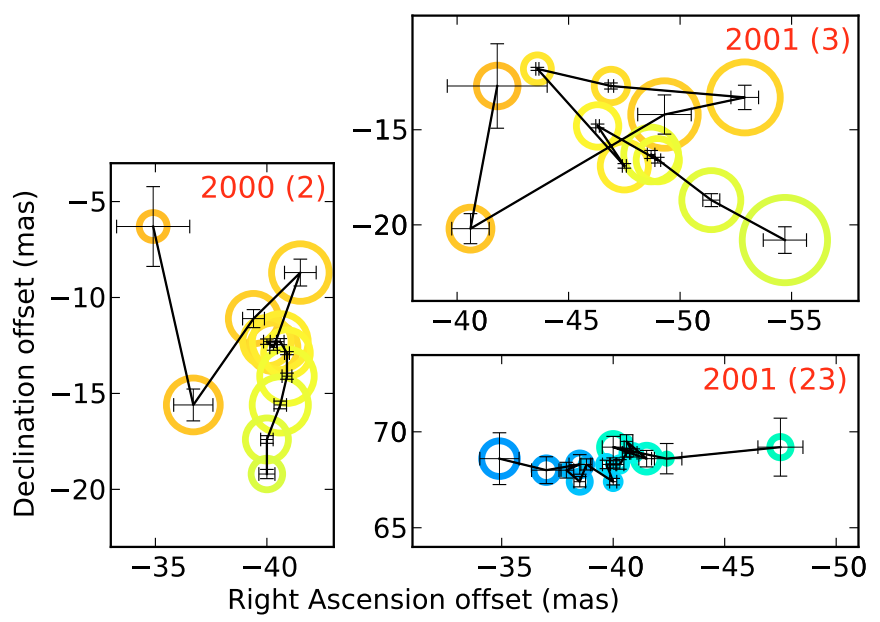

Fig. 13. The angular size and distribution of the maser components in the U Ori features marked in Fig. 12, with the same colouring scheme. Each panel shows a single feature. The diameter of each symbol is $10 \%$ of $s_{v}$ and the error bars represent the position offset uncertainties. The line joining the components shows the velocity order.

underlying assumptions; although these are large, different approaches give consistent results.

\subsubsection{Estimating the population inversion}

$\Delta n=n_{2}-n_{1}$ is the difference between the number densities per substate of the upper and lower levels involved in the maser transition, $6_{16}$ and $5_{23}$ of ortho-water (de Jong 1973). Maser amplification requires $\Delta n>0$.

We start by assuming that the emission rate is approaching the pump rate but not limited by it, following the assumptions outlined in RYC99 (Sect. 4.5 and references therein), which explains why this is reasonable, at least for the brighter masers. The population difference is given by Eq. (6.32) of Reid \& Moran (1988). RYC99 (their Eq. (16)) derived an approximation in terms of measurable quantities; omitting constants this is:

$\Delta n \propto \frac{\Delta V_{1 / 2} T_{\mathrm{B}}}{\Gamma \lambda^{2} l}$ 
A. M. S. Richards et al.: Observational evidence for the shrinking of bright maser spots

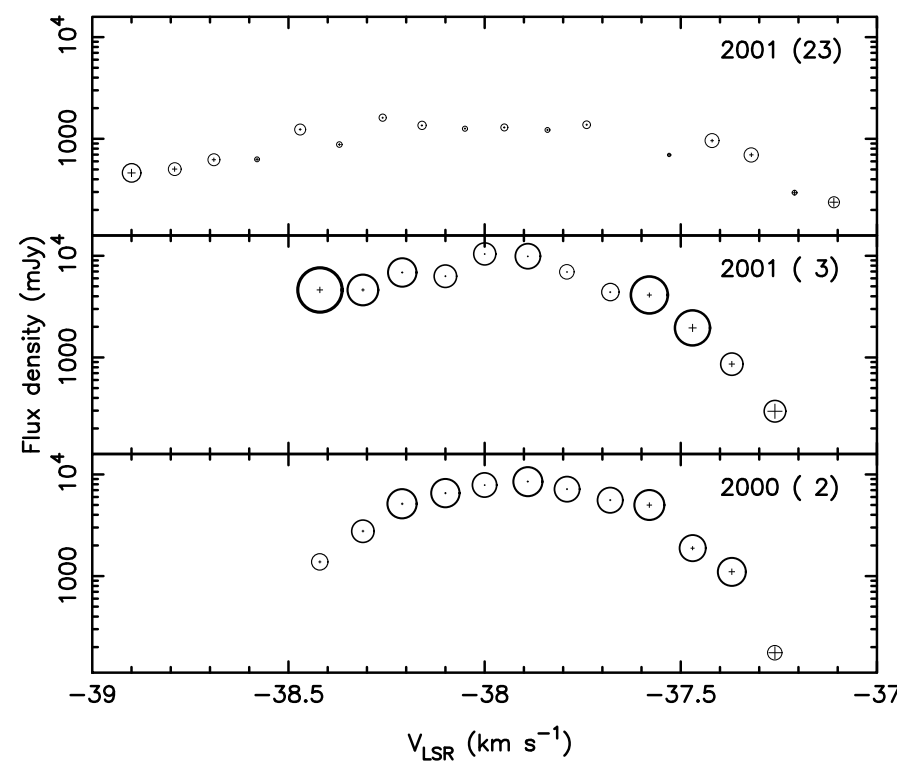

Fig. 14. The line profiles of U Ori features marked in Fig. 12. The diameters of the circles and crosses are proportional to the observed angular FWHM $s_{v}$ of the maser components and to $\sigma_{s}$, respectively.

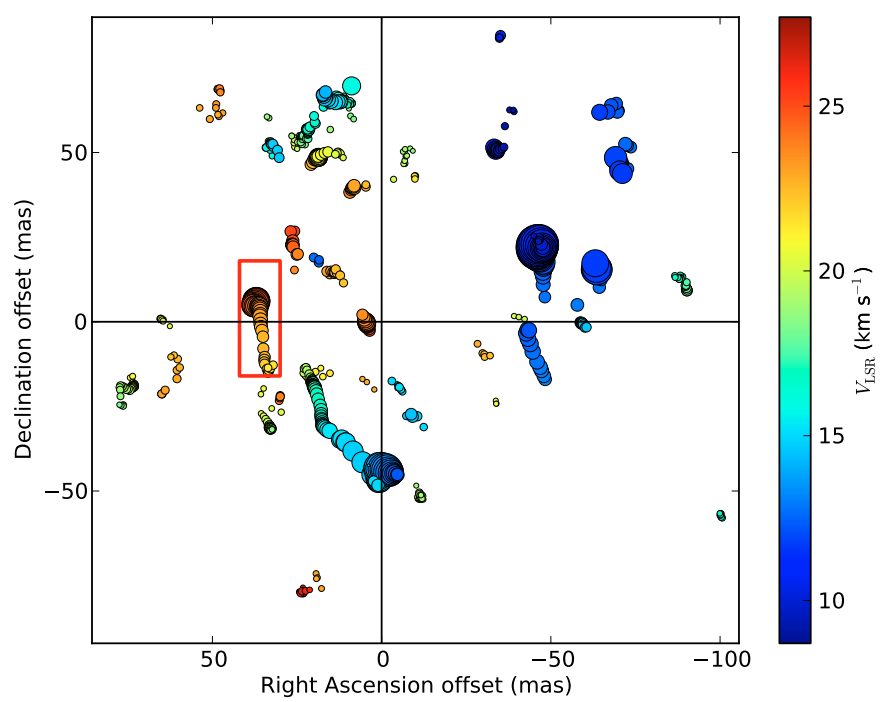

Fig. 15. Each symbol represents an $\mathrm{H}_{2} \mathrm{O}$ maser component in a single channel observed towards RT Vir, epoch 960524 . The diameter is proportional to the square root of total intensity. The colour is proportional to the velocity as shown. The boxed feature is shown in more detail in Figs. 16 and 17.

for spherical clouds. $\Gamma$ is the decay rate from the excited state, initially taken as $1 \mathrm{~s}^{-1}$ (Reid \& Moran 1988).

We obtained mean values of $10^{3} \leq \overline{\Delta n} \leq 4 \times 10^{4} \mathrm{~m}^{-3}$ $\left(0.001 \leq \overline{\Delta n} \leq 0.04 \mathrm{~cm}^{-3}\right)$ for $\mathrm{S}$ Per, U Ori, U Her and IK Tau. RT Vir had consistently high values, $10^{4} \leq \overline{\Delta n} \leq 9 \times 10^{5} \mathrm{~m}^{-3}$ $\left(0.01 \leq \overline{\Delta n} \leq 0.9 \mathrm{~cm}^{-3}\right)$.

The population of the lower state $n_{1}$ was estimated using $\left(n_{1} / n_{\mathrm{H}_{2} \mathrm{O}}\right)\left(n_{\mathrm{H}_{2} \mathrm{O}} / n_{\mathrm{H}_{2}}\right) n_{\mathrm{H}_{2}}$. We took the fractional number density of $\mathrm{H}_{2} \mathrm{O}$ with respect to $\mathrm{H}_{2}\left(n_{\mathrm{H}_{2} \mathrm{O}} / n_{\mathrm{H}_{2}}\right)$ as $2 \times 10^{-4}$, where the fraction of $\mathrm{H}_{2} \mathrm{O}$ molecules in the $n_{1}$ state is $5 \times 10^{-3}$. The actual values are likely to differ by a factor of 2 or more, both between and within sources. We take $n_{\mathrm{H}_{2}}$ as $65 \%$ of the total number density $n$, and estimate $n$ as a function of $r$ as outlined in Sect. 3.1.4.

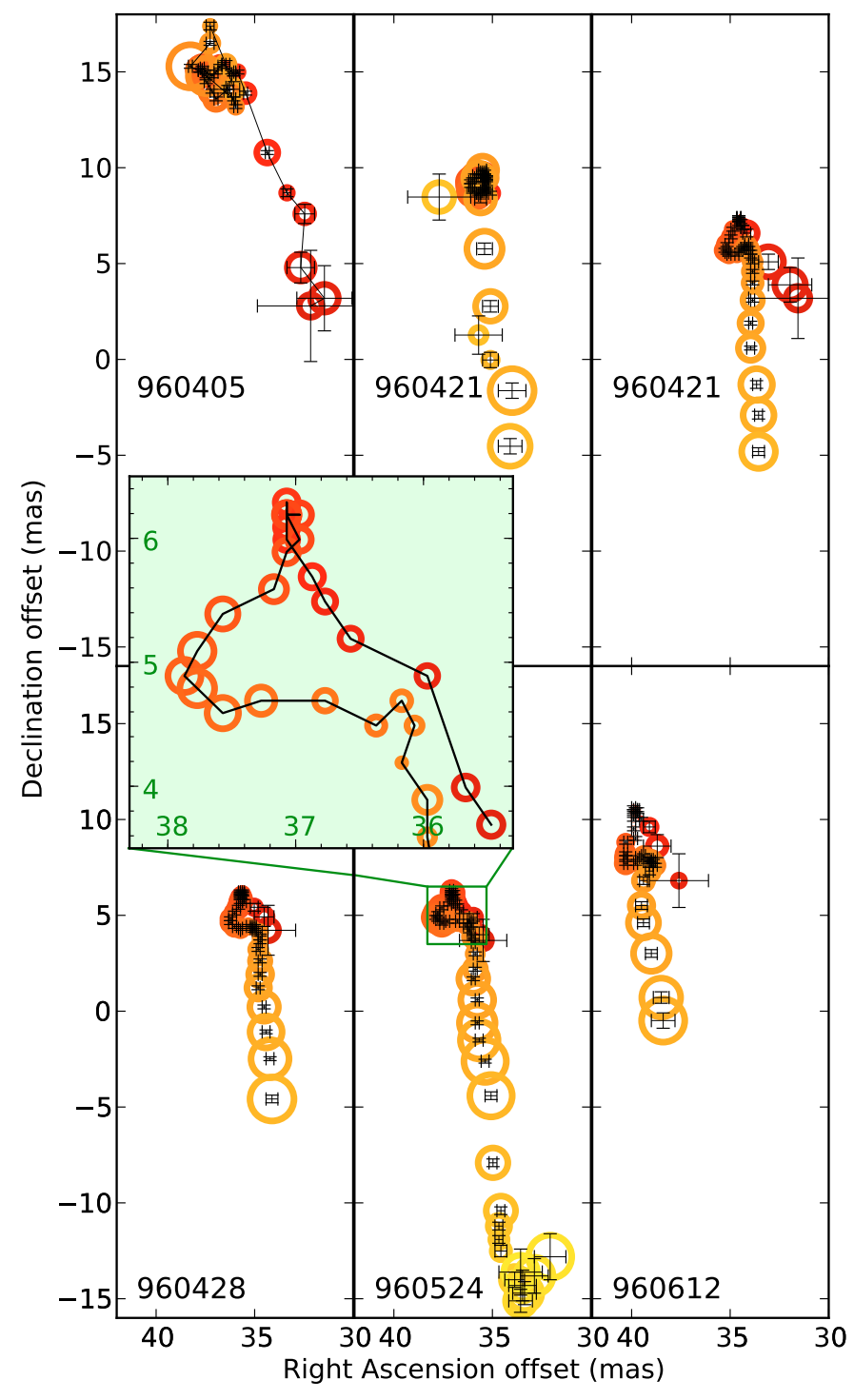

Fig. 16. The angular size and distribution of the maser components in the RT Vir feature boxed in Fig. 15, with the same colouring scheme. Each panel shows a single feature. The diameter of each symbol is $20 \%$ of $s_{v}$ and the error bars represent the position offset uncertainties. The green inset shows an enlargement of part of the 960524 feature.

If the pump rate is much higher than the stimulated emission rate, $\Delta n$ is a lower limit. If the masers are significantly saturated then the present assumptions about the geometry are affected and $\Delta n$ could be overestimated. Errors in $r$ are unlikely to exceed $30 \%$, producing less than a factor of $\sim 2$ uncertainty in $n$, and hence $n_{1} . \Delta n$ will be overestimated if $\Gamma$ or the cloud depth are underestimated. The average cloud depth is unlikely to be more than about twice $\bar{l}$ and no individual cloud depth can exceed $\sim 201$, as explained in Sect. 3.1.4. $\Gamma$ is unlikely to be that much greater than our assumed value, so $\Delta n$ is very unlikely to be more than a factor of 20 too large.

The mean percentage population inversion (weighted by measurement errors), $100 \times \Delta n /\left(n_{1}+n_{2}\right)$, was $\lesssim 1 \%$ for all sources apart from five RT Vir epochs and one IK Tau epoch which reached between $1 \%$ and $\sim 10 \%$. Selecting brighter features, with the brightness temperature at line peak $T_{\mathrm{B} 0}>10^{9} \mathrm{~K}$, gave a higher mean population inversion of a few percent for $\mathrm{S}$ Per, 
Table 3. Properties of $\mathrm{H}_{2} \mathrm{O}$ masers.

\begin{tabular}{lccccccccc}
\hline \hline Star & Date & $\overline{T_{\mathrm{B} 0}}$ & $T_{\mathrm{B} 0} \min$ & $T_{\mathrm{B} 0} \max$ & $\overline{T_{\mathrm{x}}}$ & \multicolumn{1}{c}{$\bar{\tau}$} & $\alpha$ & \multicolumn{1}{c}{$N \beta$} & $\beta$ \\
\hline S Per & 940324 & $4.5 \times 10^{11}$ & $2.6 \times 10^{06}$ & $3.5 \times 10^{13}$ & -0.2 & $9 \pm 6$ & $-1.6 \pm 0.1$ & 539 & $1.8 \pm 0.1$ \\
S Per & 990110 & $2.5 \times 10^{11}$ & $3.6 \times 10^{06}$ & $2.2 \times 10^{13}$ & -0.2 & $13 \pm 7$ & $-1.3 \pm 0.1$ & 195 & $1.0 \pm 0.1$ \\
& & & & & & & & & \\
U Ori & 940417 & $6.7 \times 10^{09}$ & $3.1 \times 10^{06}$ & $9.3 \times 10^{10}$ & -0.4 & $10 \pm 3$ & $0.6 \pm 0.1$ & 15 & $0.8 \pm 0.2$ \\
U Ori & 990109 & $2.2 \times 10^{12}$ & $2.7 \times 10^{06}$ & $2.7 \times 10^{13}$ & -0.2 & $2 \pm 5$ & $-3.1 \pm 0.2$ & 14 & $0.4 \pm 0.1$ \\
U Ori & 000410 & $7.3 \times 10^{08}$ & $2.4 \times 10^{06}$ & $2.3 \times 10^{10}$ & -1.7 & $8 \pm 3$ & $1.1 \pm 0.2$ & 6 & $0.8 \pm 0.3$ \\
U Ori & 010506 & $8.2 \times 10^{08}$ & $3.0 \times 10^{06}$ & $1.9 \times 10^{10}$ & -3.2 & $10 \pm 4$ & $1.5 \pm 0.1$ & 52 & $0.9 \pm 0.2$ \\
& & & & & & & & & \\
U Her & 940413 & $3.7 \times 10^{10}$ & $1.3 \times 10^{06}$ & $5.7 \times 10^{11}$ & -0.5 & $16 \pm 7$ & $1.6 \pm 0.1$ & 38 & $1.0 \pm 0.1$ \\
U Her & 000519 & $1.6 \times 10^{11}$ & $5.3 \times 10^{06}$ & $5.3 \times 10^{12}$ & -0.5 & $13 \pm 5$ & $2.0 \pm 0.1$ & 101 & $1.2 \pm 0.1$ \\
U Her & 010427 & $2.4 \times 10^{10}$ & $2.6 \times 10^{06}$ & $7.1 \times 10^{11}$ & -0.4 & $10 \pm 5$ & $0.8 \pm 0.1$ & 36 & $1.0 \pm 0.3$ \\
& & & & & & & & & \\
IK Tau & 940415 & $9.8 \times 10^{07}$ & $2.2 \times 10^{06}$ & $8.8 \times 10^{09}$ & -4.4 & $12 \pm 3$ & $1.2 \pm 0.1$ & 141 & $1.0 \pm 0.1$ \\
IK Tau & 000520 & $3.4 \times 10^{09}$ & $6.3 \times 10^{06}$ & $1.4 \times 10^{11}$ & -1.6 & $14 \pm 3$ & $-3.4 \pm 0.1$ & 181 & $0.8 \pm 0.1$ \\
IK Tau & 010427 & $1.0 \times 10^{10}$ & $6.0 \times 10^{06}$ & $3.7 \times 10^{11}$ & -1.2 & $14 \pm 4$ & $-2.5 \pm 0.1$ & 67 & $1.0 \pm 0.2$ \\
& & & & & & & & \\
RT Vir & 940816 & $5.1 \times 10^{09}$ & $6.2 \times 10^{06}$ & $2.0 \times 10^{11}$ & -1.4 & $17 \pm 2$ & $-1.1 \pm 0.1$ & 199 & $0.9 \pm 0.1$ \\
RT Vir & 960405 & $3.3 \times 10^{09}$ & $8.1 \times 10^{06}$ & $1.2 \times 10^{11}$ & -0.7 & $21 \pm 5$ & $-0.5 \pm 0.1$ & 106 & $0.8 \pm 0.1$ \\
RT Vir & 960421 & $3.5 \times 10^{10}$ & $1.4 \times 10^{07}$ & $8.1 \times 10^{11}$ & -0.3 & $19 \pm 4$ & $0.1 \pm 0.1$ & 116 & $0.6 \pm 0.1$ \\
RT Vir & 960429 & $1.1 \times 10^{12}$ & $1.1 \times 10^{07}$ & $5.3 \times 10^{13}$ & -0.2 & $23 \pm 5$ & $-0.8 \pm 0.1$ & 172 & $0.8 \pm 0.1$ \\
RT Vir & 960515 & $8.1 \times 10^{10}$ & $8.7 \times 10^{06}$ & $2.1 \times 10^{12}$ & -0.5 & $25 \pm 4$ & $-0.4 \pm 0.1$ & 142 & $1.0 \pm 0.1$ \\
RT Vir & 960524 & $2.5 \times 10^{10}$ & $9.4 \times 10^{06}$ & $4.4 \times 10^{11}$ & -0.6 & $25 \pm 3$ & $-0.5 \pm 0.1$ & 154 & $0.9 \pm 0.1$ \\
RT Vir & 960612 & $2.2 \times 10^{12}$ & $7.2 \times 10^{07}$ & $5.4 \times 10^{13}$ & -0.1 & $24 \pm 6$ & $-1.0 \pm 0.1$ & 100 & $0.9 \pm 0.1$ \\
\hline
\end{tabular}

Notes. All temperatures are in K. The brightness temperature $T_{\mathrm{B} 0}$ was derived using Eq. (1). $\overline{T_{\mathrm{B} 0}}$ is the mean value for the peaks of all features in each source, given along with the minimum and maximum values for the feature peaks. The mean values of the maser excitation temperature $\overline{T_{\mathrm{x}}}$ and optical depth $\bar{\tau}$ were derived as described in Sect. 3.2, which explains the uncertainties and dispersions; the given values are probably lower limits. $\alpha$ parametrises the relationship between component size and intensity for spherical, same-size clouds, as defined in Eq. (6). $N \beta$ is the number of components for which the line profile fitting was better than $3 \sigma, s_{v}>3 \sigma_{s}, s_{v}>s_{0}$ and the component lies within a feature velocity span of $\leq \Delta V_{\mathrm{D}} . \beta$ parametrises the relationship between component size, position in the line profile and the effect of the internal velocity gradient see Eq. (A.2). Numbers in italics indicate unreliable results where $<30 \%$ of the data for that epoch meet these requirements.

U Ori and U Her at most epochs with meaningful data and a few tens of percent for one IK Tau epoch and RT Vir at most epochs.

\subsubsection{Maser excitation temperature}

The maser excitation temperature is defined as

$T_{\mathrm{x}}=\left(h v / k_{\mathrm{B}}\right) / \ln \left[n_{1} /\left(\Delta n+n_{1}\right)\right]$

where $h$ and $k_{\mathrm{B}}$ are Planck's and Boltzmann's constants respectively. For $v=22.235 \mathrm{GHz}, h v / k_{\mathrm{B}} \approx 1 \mathrm{~K}$. We derived $T_{\mathrm{x}}$ at each feature peak using our values of $\Delta n$ and $n_{1}$. The error-weighted mean $\overline{T_{\mathrm{x}}}$ for each epoch (given in Table 3 ) was -5 to $-0.2 \mathrm{~K}$. $\mathrm{S}$ Per, U Her and RT Vir have $\overline{T_{\mathrm{x}}} \gtrsim-1 \mathrm{~K}$, as expected for the consistently brighter sources. The logarithmic relationship means that even if $\Delta n$ is decreased by a factor of 20 , most of the values of $\overline{T_{\mathrm{x}}}$ are decreased to not less than $-20 \mathrm{~K}$, apart from for U Ori where it approaches $-100 \mathrm{~K}$, although based on few significant measurements.

\subsubsection{Maser optical depth}

The maser optical depth for the brightest part of each cloud is related to the brightness temperature by

$\tau=\ln \left(T_{\mathrm{B} 0} /\left|T_{\mathrm{x}}\right|\right)$.

The mean $\left(\overline{T_{\mathrm{B} 0}}\right)$ and extrema of $T_{\mathrm{B} 0}$ for each source are given in Table 3. The maxima may be underestimated, if the brightest peak component size is less than the minimum resolvable size.
The numbers of features, $N F$, detected at each epoch (Table 2), do not have any significant relationship with $3 \sigma_{\text {rms }}$ (except possibly for IK Tau), suggesting that the minima are not sensitivitylimited.

We derived $\tau$ using our estimated values of $T_{\mathrm{x}}$. Table 3 gives the mean values $\bar{\tau}$ for each epoch. The dispersion includes the scatter of measured values but not the uncertainties in the underlying assumptions. $\bar{\tau}$ lies between 8 and 16 for S Per, U Her, IK Tau and the epochs of U Ori with meaningful data. RT Vir has consistently higher values reaching $\bar{\tau}=25$. If $\Delta n$ is overestimated by a factor of $20, \tau$ would be reduced by $\lesssim 5$. If $\Delta n$ is a lower limit, $T_{\mathrm{x}}$ and $\tau$ are also lower limits.

\subsubsection{Maser beaming angle}

The full angular size of a cloud and the apparent angular FWHM of maser features are approximated by $l$ and $d$ (as marked in Fig. 9). The small size of $d$ means that the relative measurement errors are large and its interpretation depends on the internal cloud structure. Figures 9, 10 and 16 show that the smallest components sampling the velocity channels near the line peak of bright features are often more closely-spaced on the sky than the fainter components in the line wings. If the maser features came from completely quiescent spheres, $d$ would be vanishingly small. We calculated the average ratio $d / s_{0}$ for each object at each epoch, where $s_{0}$ is the observed size of the single maser component closest to the peak of the line profile fitted to each feature. This ratio is in the range 0.3 to 2.4 , error-weighted mean $0.9 \pm 0.2$. This is consistent with a systematic velocity gradient 
within features of similar magnitude to the thermal velocity dispersion.

Subject to these caveats, we can obtain order-of-magnitude estimates of the beaming angle $\Omega_{\mathrm{est}}=d^{2} / l^{2}$. RYC99 obtained an average of $1.5 \pm 0.8 \times 10^{-3}$ sr for the 5 brightest features in $\mathrm{S}$ Per, which suggested that they were on the verge of saturation. We obtained error-weighted mean values per epoch, $\overline{\Omega_{\mathrm{est}}}$, in the ranges $(0.8-6) \times 10^{-3}$ sr for S Per, $(0.6-2) \times 10^{-3}$ sr for U Her and $(0.5-10) \times 10^{-3} \mathrm{sr}$ for RT Vir. U Ori and IK Tau gave larger values, of $(3-100) \times 10^{-3} \mathrm{sr}$ and $(10-50) \times 10^{-3} \mathrm{sr}$, respectively.

Vlemmings et al. (2005), following Nedoluha \& Watson (1992), show that masers remain unsaturated as long as $T_{\mathrm{B}} \Omega \leq$ $10^{10} \mathrm{~K} \mathrm{sr}$, for $\Gamma=1 \mathrm{~s}^{-1}$. We obtain average values of $\overline{T_{\mathrm{B} 0} \Omega_{\mathrm{est}}}<10^{9} \mathrm{~K}$ sr for all sources apart from RT Vir, where $\overline{T_{\mathrm{B} 0} \Omega_{\mathrm{est}}} \lesssim 10^{11} \mathrm{~K}$ sr. However, the rapid variability of RT Vir (Richards et al. 1999b; Lekht et al. 1999) suggests that its masers are also largely unsaturated, possible if $\Gamma>1$. Vlemmings \& van Langevelde (2005) estimate $T_{\mathrm{B}} \Omega \approx 10^{10}-10^{11}$ for $\mathrm{S}$ Per from line profile analysis and deduce that the masers are mostly unsaturated, although their VLBA observations only detected the brighter, more compact emission.

\subsubsection{Maser line narrowing}

The maser FWHM linewidth is given by $\Delta V_{1 / 2}=\Delta V_{\mathrm{D}} / \sqrt{1+\tau}$ (Elitzur 1992) for unsaturated amplification of ambient thermal radiation. This predicts $0.25 \lesssim \Delta V_{1 / 2} \lesssim 0.4$ for $\tau$ in the range shown in Table 3 and $\Delta V_{\mathrm{D}}$ between $1.2-1.6 \mathrm{~km} \mathrm{~s}^{-1}$. The average measured values of $\Delta V_{1 / 2}$, given in Table 2, are greater than predicted, whilst narrower than $\Delta V_{\mathrm{D}}$. Approaching saturation causes the line profile to re-broaden. Alternatively, the observed linewidths may be explained by the velocity gradients within the clouds. Vlemmings \& van Langevelde (2005) show that, in the presence of a velocity difference along the maser path of up to $\Delta V_{\mathrm{D}}$, the unsaturated maser line width is up to about double the width at constant velocity.

\subsubsection{Predominantly unsaturated masers}

A large value of $\Delta n$ provides stronger population inversion which provides conditions for bright, unsaturated emission (see Reid \& Moran 1988, their Fig. 6.2), and a negative $T_{\mathrm{x}}$ of small magnitude, approaching zero from below. However, if the maser approaches saturation, $\Delta n$ becomes small and $T_{\mathrm{x}}$ approaches $-\infty$ as the maser emission rate becomes close to the pump rate. The small-magnitude negative values we obtained for $T_{\mathrm{x}}$ suggest that even the most intense $\mathrm{H}_{2} \mathrm{O}$ masers in these objects are unlikely to be strongly saturated. Our estimates of beaming angle and line narrowing also rule out strongly saturated water masers.

We investigated the relationship between excitation temperature and peak brightness temperature for each source by evaluating the slope $A$ of the expression $\log \left|T_{\mathrm{x}}\right| \propto A \log T_{\mathrm{B} 0}$, for measurements more significant than than $3 \sigma$. This is an inverse relationship at every epoch. RT Vir has the shallowest slopes, averaging $-0.6 \pm 0.2$; the average slopes for the other sources are U Her $-0.8 \pm 0.1, S$ Per $-0.9 \pm 0.1$ and IK Tau $-1.0 \pm 0.2$. U Ori has the least significant data, giving $-0.6 \pm 0.5$. This shows that the brightest masers are associated with the smallest-magnitude values of $T_{\mathrm{x}}$, consistent with unsaturated emission.

The maser optical depth $\tau$ is equivalent to the product of the unsaturated absorption coefficient $\kappa_{0}$ and the path length $l$.

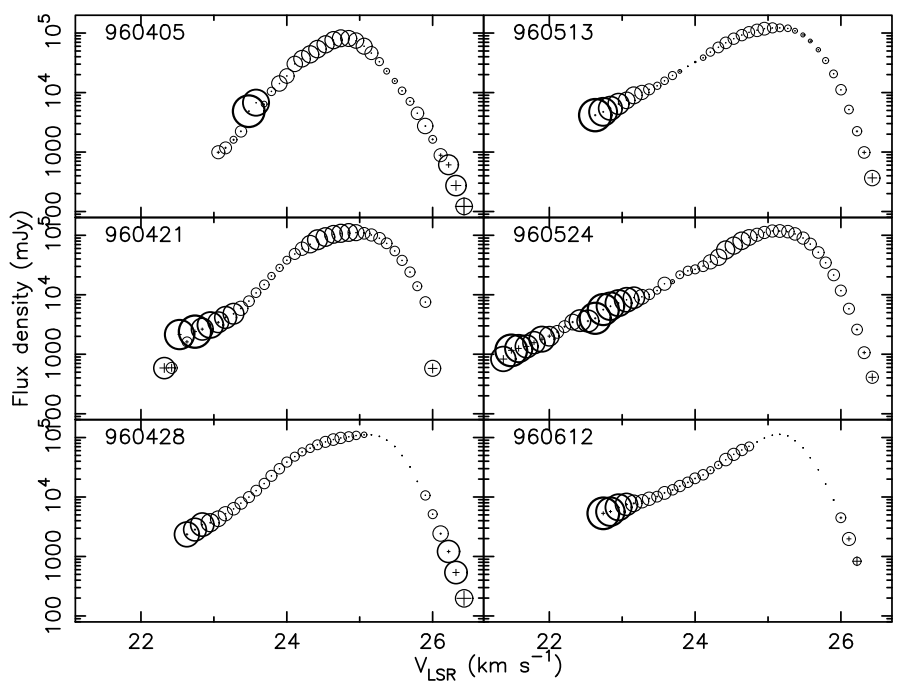

Fig. 17. The line profiles of RT Vir features marked in Fig. 15. The diameters of the circles and crosses are proportional to the observed angular FWHM $s_{v}$ of the maser components and to $\sigma_{s}$, respectively.

We can deduce from the arguments of EHM $92^{2}$ that a spherical maser begins to saturate when $\tau \gtrsim 17$ and becomes fully saturated when $\tau \gtrsim 100$. The values of $\tau$ which we obtain are well within the unsaturated or slightly saturated regimes. All these results imply that circumstellar $22-\mathrm{GHz}_{2} \mathrm{O}$ masers are predominantly unsaturated.

\section{The observed size of maser components}

The brightest components near the line centres have more compact sizes in many of our sources, with larger components in the line wings. Figures 3-7 show the variations of $s_{v}$ across the line profiles at each epoch. $s_{v}$ increases significantly from the line centres to the wings of almost all S Per features (apart from the faintest, with larger uncertainties). Most RT Vir features also show an inverse variation of $s_{v}$ with intensity, although some components near the peaks are anomalously swollen. In contrast, many U Ori and U Her features have no apparent correlation between $s_{v}$ and intensity. IK Tau shows a mixture of behaviours. Figures 9, 11, 14 and 17 show the line profiles of the selected features labelled in Figs. 8, 12 and 15. These are examples of differences in appearance which are characteristic of the three sources. U Her is similar to U Ori and IK Tau is somewhat similar to RT Vir.

Figures 10, 13 and 16 show $s_{v}$ as a function of position for the selected features (at $20 \%$ of the actual measured size of $s_{v}$, in order to avoid excessive overlaps). The error bars represent the position errors. These plots show that $s_{v} / l$ ranges from $\ll 1 \%$ for the peak S Per and RT Vir components, to $>50 \%$ for the components close to their line edges, whilst U Ori shows a less ordered scatter.

In the following sections, we compare the observed relationships between the sizes of individual maser components and their intensities, with the models developed by EHM92. We tentatively investigate the effects of the sizes and velocity gradients of the parent clouds. A fully quantitative analysis is difficult due to the difficulties in measuring the three-dimensional structure of the clouds nor of the beaming angle. Nonetheless, we

2 Note that EHM92 use $l$ as the radius of a cloud whereas we use it as the diameter. 
are able to distinguish between the predictions for amplificationand matter-bounded masers and identify which aspects of maser behaviour are well-described by current theories and which confound them.

\subsection{Spherical, uniform clouds}

We start by comparing our results with the simplest model, for spherical, density bounded clouds. EHM92 show that these would produce amplification-bounded masers wherein the beaming angle should increase with frequency offset from the line centre. Initially, we assume that all the clouds are the same size and all are spherical (i.e., that $l$ is constant within each source).

\subsubsection{Component size variation with intensity: the model}

The observed size $s_{v}$ represents the angular FWHM of the distribution of the intensity of maser emission from water molecules in a particular cloud in the velocity interval sampled by a single channel. Using expressions from Elitzur (1992) for the effect of the maser amplification process in spherical clouds, the beaming solid angle is given by $\Omega=\pi / 0.5 l \kappa_{0 v}$, for unsaturated emission from a sphere of diameter $l$, where the unsaturated absorption coefficient for that velocity channel is $\kappa_{0 v}$. (If the emission is significantly saturated the beaming angle is reduced by a factor of $r_{s} / 0.5 l$ where $r_{s}$ is the radius of the unsaturated core.) The observed area is given by $A=(\pi / 4 \ln 2) s_{v}^{2}=\Omega(0.5 l)^{2}$, so the apparent (unsaturated) component size should be given by

$s_{v}^{2} \propto 0.5 l / \kappa_{0 v}$.

The intensity of unsaturated emission from a single velocity channel though a cloud of depth $l$ is given by $I \approx S_{0} \mathrm{e}^{K_{0 v} l}$, if $I \gg S_{0}$, the input radiation (generally the ambient local thermal radiation at $22 \mathrm{GHz}$ ). This is clearly the case in CSEs where the background radiation is undetectable by interferometric observations on the scale of the masers. The stellar continuum could be amplified by a cloud along the line of sight, but there is no evidence for exceptionally bright blue-shifted peaks in our data. The volume filling factor is $\$ 1.5 \%$ so cloud overlap is not likely to be significant. The maser component size is related to the emission intensity by $I / S_{0} \propto \mathrm{e}^{0.5 l^{2} / s_{v}^{2}}$ so (assuming $\ln I \gg \ln S_{0}$ )

$\log (s) \propto \log (\sqrt{0.5} l)+\alpha \log (\ln I)$.

\subsubsection{Relationships between observed component size and intensity}

We test the simple, spherical model for amplification-bounded masers (from clouds of constant size) by plotting $\log s$ against $\log (\ln I)$. Equation (6) predicts that this should give a slope of $\alpha=-0.5$. Figures $18-23$ show least-squares error-weighted fits to the data for all sources. The values of $\alpha$ are given in Table 3 . $\alpha$ is negative, showing that the apparent component size shrinks with increasing intensity, at both epochs for S Per, at 6/7 epochs for RT Vir, at 2/3 epochs for IK Tau and at 1/4 epochs for U Ori.

Figures 22 and 23 show that RT Vir most closely exhibits the behaviour predicted for spherical clouds, with a mean value of $\alpha$ of -0.5 for all 7 epochs. The behaviour is fairly consistent between epochs.

S Per shows $\alpha<-0.5$ for both epochs, as plotted in Fig. 18. The uncertainty in $s_{v}$ is inversely proportional to $I$ and hence

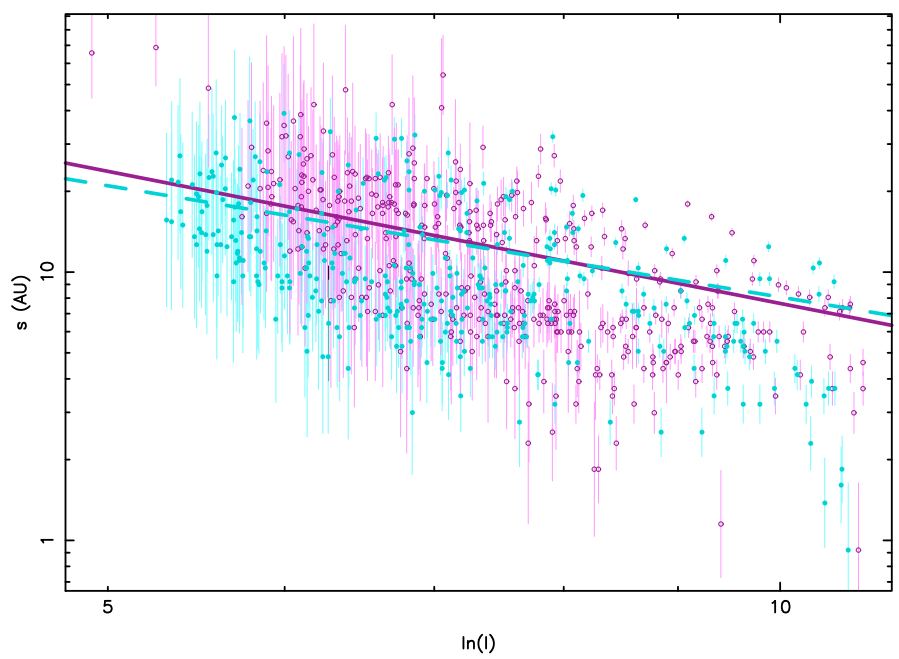

Fig. 18. S Per maser component FWHM $s_{v}$ as a function of the natural logarithm of the intensity $I$ coded per epoch as in Fig. 19.

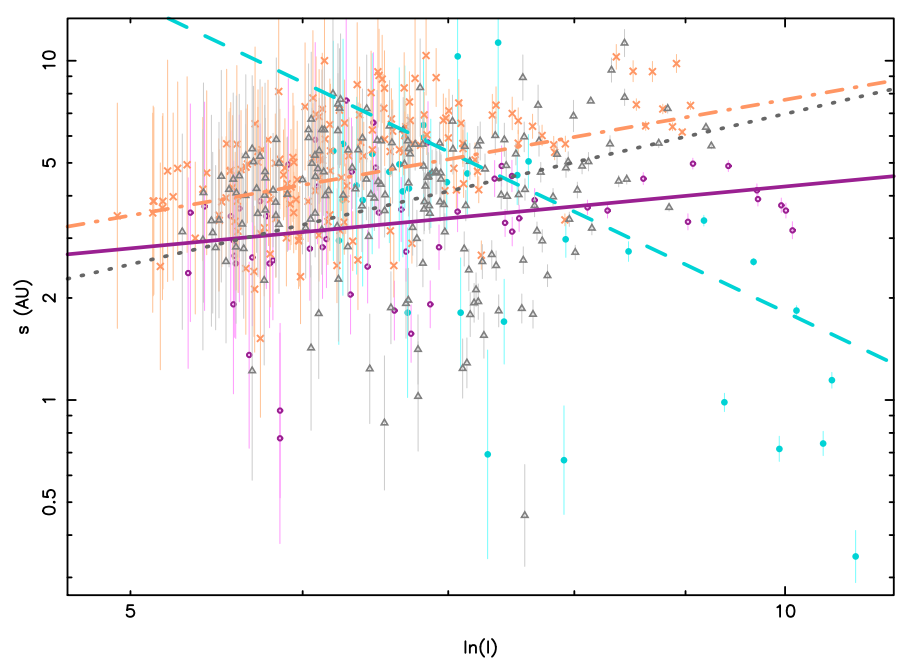

Fig. 19. U Ori maser component FWHM $s_{v}$ as a function of the natural logarithm of the intensity $I$, for components where $s_{v}>3 \sigma_{s}$. The magenta (hollow), cyan (solid), orange (cross) and grey (triangle) symbols represent 1994, 1999, 2000 and 2001 results, respectively. The corresponding error-weighted least-squares fits (to all data) are shown as solid, dashed, dot-dashed and dotted lines.

$\sigma_{s}$ is greater for fainter components, biasing the fit towards the contribution of the brighter components. These are most likely to be approaching saturation, causing a steeper shrinkage of $\Omega$ with increasing maser amplification. There are sufficient data for $\mathrm{S}$ Per to examine the properties for subsets. Selecting the fainter components only, with $I<0.6 \mathrm{Jy}$, gives $\alpha=-0.6$ and -0.4 for the 1994 and 1999 data respectively, close to the predicted values for unsaturated emission. This suggests that the steeper slopes measured for the whole data sets are due to saturation of the brighter components. This is consistent with the conclusion of RYC99 that the brightest features are approaching saturation.

IK Tau also produces $\alpha<-0.5$ in 2000 and 2001, but inspection of Fig. 21 suggests a similar explanation as for S Per, although attempting to fit to sub-sets of the data produces a very large scatter. The apparent slope of $\alpha>0$ in 1994 is due to a small group of bright components showing anomalous behaviour; if these are excluded by selecting $I<7.5 \mathrm{Jy}$, a slope of -1.6 is obtained, whilst for $I<0.75 \mathrm{Jy}, \alpha=-0.6$, but with low 


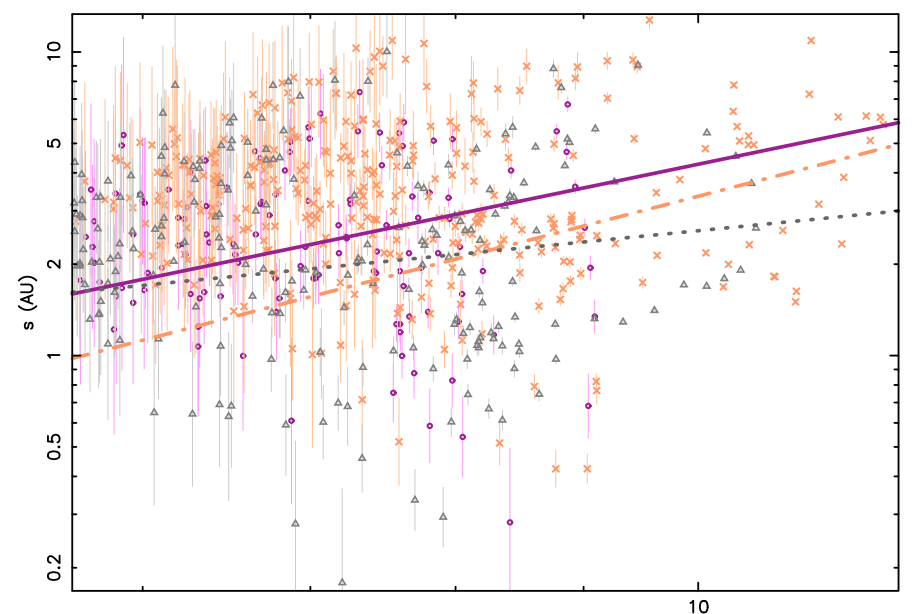

$\ln (1)$

Fig. 20. U Her maser component FWHM $s_{v}$ as a function of the natural logarithm of the intensity $I$, coded per epoch as in Fig. 19.

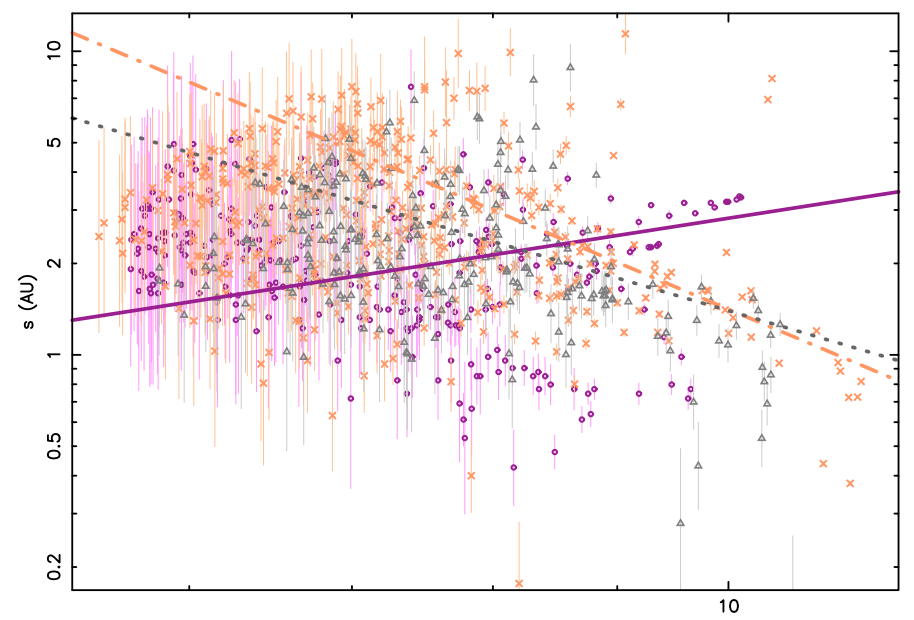

$\ln (1)$

Fig. 21. IK Tau maser component FWHM $s_{v}$ as a function of the natural logarithm of the intensity $I$, coded per epoch as in Fig. 19.

significance since there are few points with accurate measurements.

$\mathrm{U}$ Ori and U Her have $\alpha>0$ for all but one epoch and Figs. 19 and 20 show very large scatters. This shows that most of their maser regions do not behave as spherical clouds, discussed further in Sect. 5.2.

\subsection{Cloud size variations and velocity gradients}

The observed size of clouds within each CSE varies by a factor of two or more (Table 2). We investigated whether this can be included meaningfully in our models and attempted to allow for the effects of velocity gradients within clouds. This is described in Appendix A. In brief, we modified Eq. (6) to include the change in path length through a cloud with velocity offset. Fitting this to the data gave slopes roughly consistent with the expected relationship between component size and position in the line profile, for those sources which showed the behaviour expected from spherical clouds (i.e. $\alpha<0$ in Eq. (6)). However there were very large scatters, unsurprising because we are measuring velocity along the line of sight but angular separation perpendicular to this. In reality the clouds are not perfectly

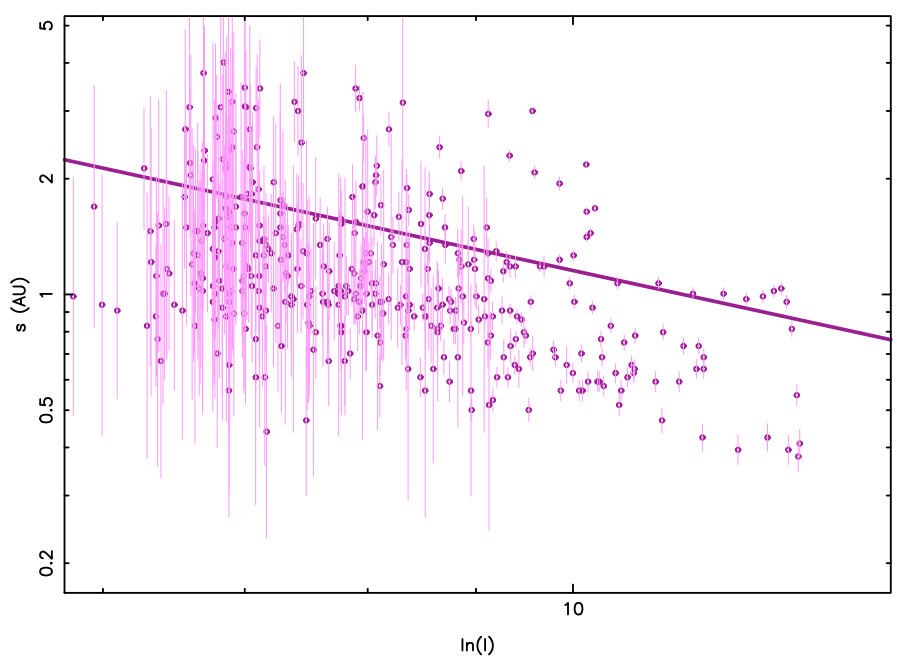

Fig. 22. RT Vir maser component FWHM $s_{v}$ as a function of the natural logarithm of the intensity $I$, observed in 1994.

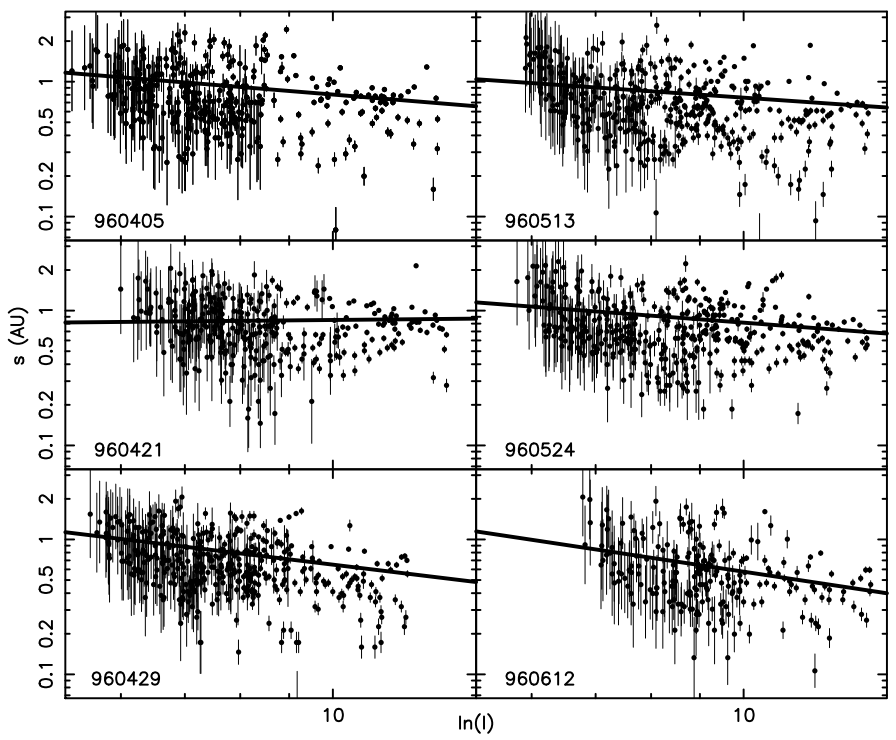

Fig. 23. RT Vir maser component FWHM $s_{v}$ as a function of the natural logarithm of the intensity $I$, observed in 1996.

spherically symmetric and we have insufficient constraints on the three dimensional structure of individual clouds to improve these results. Nonetheless, insofar as the results in Appendix A are meaningful, they are consistent with the results in Sect. 4.1.2.

\subsection{Confirmation and limitations of the predictions for amplification-bounded masing}

To sum up these results, the measured size of maser components has an inverse relationship with intensity (Sect. 4.1) for S Per, RT Vir and most epochs of IK Tau, as predicted for amplification-bounded masers. In some of these cases, the component size shrinks more steeply than expected. This is partly because results are biased towards the brightest components, which may be approaching saturation. The remaining sources, $\mathrm{U}$ Ori and U Her, show a mixture of behaviours and may be matter-bounded, see Sect. 5. This first, simple investigation, of the relationship between component size and intensity, assumed that all the features in a given source/epoch arise from identical, quiescent spheres. 
We next attempted to investigate the behaviour of components within features, with respect to the component at the peak of each line, for masers meeting the amplification-bounded criterion of $s v>s_{0}$ (Appendix A). We derived a semi-empirical relationship which included the effects of velocity gradients. The average ratio of the size of a component with respect to the size of the line peak component does increase with displacement from the line centre, but more weakly than predicted.

EHM92 note that the beaming angle has a linear (rather than quadratic) dependence on the ratio between the observed size and the depth of an emission region, when these are determined by orthogonal velocity gradients. However, further work is needed to explore application of this model quantitatively to our data due to the probable unsaturated state and irregular velocity gradients. In future, we hope to use proper motion studies which could establish the relationship between the velocity gradients parallel and perpendicular to the line of sight. $e$-MERLIN, will have at least double the sensitivity of MERLIN in the same width velocity channel, and allow sampling of any velocity span at any desirable resolution.

\section{Beaming and the properties of masing clouds}

We have compared maser properties with the predictions of maser beaming models. We now examine the implications for the geometry of the individual masing features and the conditions in the CSEs as a whole. Table 2 shows that $\epsilon \geq 0.5$, and $r_{\mathrm{o}} \sim(3-4) r_{\mathrm{i}}$. Applying the expressions given in Sect. 3.1.4 suggests that if clouds are initially spherical, expanding radially away from the central star under radiation pressure alone, their tangential:radial aspect ratio will remain $<2$. Some of our results are consistent with this scenario (Sect. 5.1) but in other sources some of the clouds may be more flattened (Sect. 5.2).

\subsection{Amplification-bounded masers}

The measured size $s_{v}$ of $\mathrm{H}_{2} \mathrm{O}$ maser components decreases with increasing maser intensity, for emission from $\mathrm{S}$ Per, and, for the majority of epochs, from RT Vir and IK Tau (Sect. 4). The relationships roughly follow the expected relationship for maser beaming from unsaturated, spherical masers (Eq. (6)). EHM92 call such masers "amplification bounded" since the amplification process controls the observed size.

The beaming angle should increase with the frequency offset from line centre within an amplification-bounded maser feature. The relative observed component size, $\ln \left(s_{v} / s_{0}\right)$, is expected to be proportional to the velocity displacement from the line peak; we also attempt to allow for the observed velocity gradients using a semi-empirical relationship (Eq. (A.2)). Qualitative agreements are obtained for sources/epochs where amplification-bounded masing dominates but the slopes are shallower than predicted, probably due to the over-simplified nature of the model and the non-linear velocity gradients.

One aspect of the behaviour of RT Vir is anomalous. Figures 7 and 17 show that, in some of the brightest clouds, the components in the line wings appear relatively large, shrinking at first as expected towards the centre of the line, but then the observed sizes re-broaden near the peaks of the spectral features. This behaviour is very variable, e.g. the smallest components of the bright feature at $11-13 \mathrm{~km} \mathrm{~s}^{-1}$ appear on the near side of the peak with respect to $V_{\star}$ (Fig. 7) at epoch 1996a, but are located on the far side at epochs just a few weeks later. The peak velocity with respect to $V_{\star}$ increases by $\gtrsim 1 \mathrm{~km} \mathrm{~s}^{-1}$ over this period.
IK Tau also shows hints of such behaviour in Fig. 6. This spatial re-broadening cannot be explained by current maser theory.

\subsection{Matter-bounded masers}

The other two AGB stars, U Her and U Ori, and some IK Tau features, show a very loose relationship between $s_{v}$ and $I$. Inspection of their velocity profiles (Figs. 4 and 5) shows that the components in some bright features at some epochs have the inverse relationship between observed size and intensity expected for spherical clouds. The components in other features, however, show very little variation with intensity, and some brighter features exhibit larger components than do fainter features at the same epoch. EHM92 explain how, in "matter-bounded" beaming, the measured maser size is expected to be independent of frequency, i.e. location in the line profile. This is also consistent with the larger values of $\Omega_{\text {est }}$ obtained for $U$ Ori than for any other source, although these have large uncertainties (Sect. 3.2.4).

Matter-bounded masers emanate from regions where the amplification path along the line of sight is longer than one or both physical dimensions of the emitting region in the plane of the sky. EHM92 develop a model for maser amplification through a flattened cloud perpendicular to the shock direction using disclike masers (resembling a coin viewed edge-on), and filamentary masers, which can be envisaged as narrow tubes of amplification pointing at the observer. They explain how the apparent size of such masers remains similar to the true smaller dimension of the emission region in the plane of the sky, with little dependence on intensity or position in the line profile.

Figure 2 shows that U Her and U Ori have a higher proportion of larger components than the other AGB stars, with the peaks of the distribution of $s_{v}$ comparable to $l$ at some epochs. EHM92 predicted that filamentary masers would exhibit an irregular distribution of components whilst a more linear distribution is expected for disc masers. The distribution of maser components in some features around U Ori (Figs. 12 and 13) and $\mathrm{U}$ Her is indeed scattered, although some of the features which show an increase of component size towards the line peak do have apparently linear structures, possibly due to velocity gradients.

From EHM92 Eq. (2.2), a masing region can behave as a filamentary maser if its depth along the line of sight ( $2 l$ in the notation of EHM92) exceeds $\sqrt{\tau}$ times its width in the perpendicular direction ( $2 h$ in the notation of EHM92). U Her and U Ori have $\tau \lesssim 16$ (Table 3 ), derived by assuming spherical clouds. $\tau$ has an inverse logarithmic dependence on $\left|T_{x}\right|$, which in turn has a logarithmic dependence on cloud depth (as explained in Sect. 3.2.3). Hence, if the depths of some clouds along the line of sight is greater than the measured size $l, \tau$ will be slightly reduced.

Taking this into account, the filamentary condition requires maser clouds with a depth $\sim 5$ times their width. If we take $\bar{l}$ as the width, this implies cloud depths of 12-25 AU for U Her and $\mathrm{U}$ Ori, which is only half the maser shell depth $\left(r_{\mathrm{o}}-r_{\mathrm{i}}\right)$. We do see some very long component series, e.g. the feature running from $(-20,-20)$ to $(-10,-60)$ in Fig. 12 epoch 1994, about 10 AU in extent. This may be due to the effect of a velocity gradient which allows us to see many components across the narrow edge of a disc maser.

If clouds are initially spherical, but develop an aspect ratio $\approx 5$, this cannot be achieved by radiation pressure alone, as explained at the start of this section. The other possible cause is shock compression and U Her and U Ori are in fact the most 
likely candidates for strong shocks reaching the $\mathrm{H}_{2} \mathrm{O}$ maser region.

The $\mathrm{H}_{2} \mathrm{O}$ maser shells around $\mathrm{U}$ Ori and $\mathrm{U}$ Her have volume filling factors 2-3 times smaller than around RT Vir and IK Tau, despite having a higher mass loss rate than RT Vir, which implies that masing clouds are more disrupted around U Ori and U Her. Samus et al. (2008) and data from the AAVSO (American association of variable star observers) show that U Ori and U Her have regular, deep stellar pulsations with amplitudes $\approx 5$ mag, but IK Tau is a somewhat less regular pulsator, with erratic amplitudes. S Per and RT Vir are semi-regular variables, with pulsation amplitudes of $<3$ and $<1 \mathrm{mag}$, respectively. Stronger shocks are likely to propagate out from the stellar surfaces of U Ori and U Her, which could flatten maser clouds. Rudnitskij et al. (2000) describes signs of the impact of stellar pulsations apparent from long-term spectral monitoring of $\mathrm{H}_{2} \mathrm{O}$ masers around U Ori. Moreover, both U Ori and U Her have undergone $\mathrm{OH}$ maser flares likely to have originated in the inner CSE (Pataki \& Kolena 1974; Chapman \& Cohen 1985; Etoka \& Le Squeren 1997).

\section{Conclusions and summary}

We measured the properties of $\mathrm{H}_{2} \mathrm{O}$ masers around $\mathrm{S}$ Per, U Ori, U Her, IK Tau and RT Vir at multiple epochs using MERLIN. The emission in each 0.1 or $0.2 \mathrm{~km} \mathrm{~s}^{-1}$ velocity channel is resolved into milli-arcsec scale components, which form series in sucessive channels generally corresponding to a single spectral feature with a Gaussian-like profile. Many of these features have systematic position-velocity gradients which allow us to estimate the physical extent of maser clouds. The number densities required for masing and other properties show that the clouds are probably discrete physical entities. The average feature peak brightness temperatures are $10^{8} \lesssim \overline{T_{\mathrm{b}}} \lesssim 10^{12} \mathrm{~K}$.

Most of the maser features have negative excitation temperatures close to zero and modest optical depths, consistent with mainly unsaturated masers. The excess population of the upper level of the maser transition is $<1 \%$ of the combined number density of both states, except for RT Vir, where it reaches $\sim 10 \%$ at some epochs. We use this to infer maser excitation temperatures of $-0.1 \gtrsim \overline{T_{\mathrm{x}}} \gtrsim-5 \mathrm{~K}$. This leads to optical depths of 8-25 (excluding two epochs with insufficient good data).

We find an inverse relationship between the measurable size of maser components in individual velocity channels and the intensity, for S Per and for all but one epoch each of IK Tau and RT Vir. This is broadly consistent with the relationship predicted by EHM92, $s_{v} \propto(\ln I)^{-0.5}$, for amplification-bounded masing from uniform, spherical clouds. The brightest $\mathrm{H}_{2} \mathrm{O}$ components around S Per and IK Tau shrink more steeply than predicted with increasing intensity, suggesting that they are partially saturated. In such conditions the effective observed size is reduced proportionally to the size of the unsaturated core. The relationship between the relative size of each component and its position in the line profile of amplification-bounded masers is expected to obey $\ln \left(s_{v} / s_{0}\right) \propto 1 / 2\left(\delta v / \Delta V_{\mathrm{D}}\right)^{2}$ (where $s_{0}$ is the size of the peak component in each feature and $s_{v}$ is the size of the component at velocity offset $\delta v)$. We find that $\ln \left(s_{v} / s_{0}\right)$ has a weaker relationship with $\delta v$. Inspection of the plots of the variation of the RT Vir component sizes across its line profiles shows that the brightest components re-broaden noticeably, which cannot be explained.

Most U Ori and U Her features and some around IK Tau show contrasting behaviour more suggestive of matter-bounded masers. The size of maser components is almost independent of $\ln I$, being generally larger in proportion to the sizes of features (l). EHM92 predicted that observed component size is independent of position in the line profile for matter-bounded masing. Many features in U Her and U Ori are likely to meet the filamentary condition, due to disc-like masers, with an aspect ratio $\$ 5$. The components of U Her and U Ori have larger observed sizes than for the other two AGB stars, and U Ori has the largest beaming angles. The random spatial distribution of components within some U Ori and U Her features is also consistent with the predictions of EHM92 for matter-bounded masers. These sources have the deepest and most regular pulsation periods, so shocks could propagate more strongly into the $\mathrm{H}_{2} \mathrm{O}$ maser shell and flatten the clouds.

The two scenarios, for amplification- and matter-bounded masers, are compared in a cartoon in Fig. 1. The upper diagrams show how maser propagation through a sphere will give a very narrow apparent spatial size at full width half maximum (left) but propagation down the long axis of a disc (right) produces an apparent size more similar to that of the emission region. The star names are in order of the degree to which they present the type of masing behaviour. These high-resolution MERLIN observations provide direct observational tests of the predictions of EHM92. Most circumstellar 22- $\mathrm{GHz} \mathrm{H}_{2} \mathrm{O}$ masers behave as spherical, amplification-bounded clouds. They are probably unsaturated, the warm conditions and small size (relative to starforming regions) providing a very efficient pump which allows them to reach high maser optical depths. Some of the maser features round the Mira variables with the deepest-amplitude pulsations appear to be matter-bounded, possibly emanating from shock-flattened clouds.

Acknowledgements. We thank Indra Bains, Wouter Vlemmings and the University of Manchester Stellar group for stimulating discussions. We also thank Bains, Alexios Louridas and Daniel Rosa-Gonzales for the use of the data from RYC99 and B+03. We acknowledge with thanks the variable star observations from the AAVSO International Database contributed by observers worldwide and used in this research. M.E. acknowledges the support of NSF grant AST-0807417. We are very grateful to the referee, whose careful inspection of the manuscript led to great improvements in clarity.

\section{Appendix A: Cloud size variations and velocity gradients}

The observed size of clouds within each CSE varies by a factor of two or more (Table 2). This Appendix investigates whether this can be included meaningfully in our models, followed by an attempt to allow for the effect of velocity gradients. The end results are qualitatively consistent with Sect. 4.1.2 but the unknown, probably irregular, shapes of the clouds means that we cannot obtain precise measurements.

The effect of variations in size of spherical clouds (with purely thermal velocity dispersions) was tested by rearranging Eq. (6) in order to plot $\log s v / l$ against $\log (\ln I)$. We obtained slopes similar to the values of $\alpha$ given in Table 3, or slightly steeper. All S Per, IK Tau and RT Vir epochs had negative slopes, averages $-1.3,-1.4$ and -0.9 , respectively; U Her and U Ori had large scatters with positive averages. These results were of lower significance than the evaluation of $\alpha$ described in Sect. 4.1.2. It seems that additional effects are more significant than variations in cloud size, in producing deviations from the predicted value of $\alpha=-0.5$. This is not surprising, since the presence of velocity gradients is what enables us to obtain values of $l$ close to the physical size of clouds.

We tried a different approach by starting with an expression predicting the variation of component size with intensity as a 
function of velocity offset from the peak of the line profile for each cloud. Initially we retain a purely thermal velocity dispersion as we assume that all clouds are in the same saturation state in a given source. The ratio of the measured size of a maser component in any velocity channel, to the size of the component at the line peak, $s_{v} / s_{0}$, should then be a consistent function of velocity offset from the line centre. We take $s_{0}$ as the size of the component closest in velocity to the centre of the spectral profile fitted to each feature as described in Sect. 2.

Following E92, the unsaturated absorption coefficient varies across the line profile of amplification-bounded masers as $\kappa_{0 v} / \kappa_{00}=\mathrm{e}^{-\left(\delta v / \Delta V_{\mathrm{D}}\right)^{2}}$ where the absorption coefficient is $\kappa_{00}$ at the line centre, velocity $V_{0}$, and $\kappa_{0 v}$ is the absorption coefficient at velocity offset $\delta v$. The thermal line width is a weak function of distance from the star, $\Delta V_{\mathrm{D}} \propto r^{-0.2}$ (Sect. 3.1.1). We estimated $r$ by solving for the 3D structure of each $\mathrm{H}_{2} \mathrm{O}$ maser shell as described in Murakawa et al. (2003).

Using these relationships in Eq. (5) gives an expression for $s_{v}$ as a function of location in the line profile

$\ln \left(\frac{s_{v}}{s_{0}}\right) \propto \frac{1}{2}\left(\frac{\delta v}{r^{-0.2}}\right)^{2}$.

However, testing this relationship on our data did not show the expected quadratic dependence but a much weaker exponent, between 0 and 1 if it was meaningful at all.

We now attempt to extend known analytic relationships in order to investigate an empirical relationship describing the observed maser beaming by including the effects of velocity gradients in Eq. (A.1).

Figures 8 and 15 show that many clouds possess systematic gradients of velocity with position. The features in Figs. 10A and B and 16 present orderly but non-linear structure. The progression from channel to channel of component size and location can be followed clearly despite twists and turns (see RT Vir inset). Many features in IK Tau and a few in U Her and U Ori also have systematic gradients although the less ordered distributions seen in Figs. 12 and 13 are more typical of U Ori and $\mathrm{U}$ Her. The total velocity spans $(\Delta V)$ of features are similar in all sources and the ratio $\Delta V / l$ is about $0.1-0.2 \mathrm{~km} \mathrm{~s}^{-1} \mathrm{AU}^{-1}$ for S Per (RYC99), and an order of magnitude greater for the smaller AGB clouds $(B+03)$.

The angular separations between components within a cloud, especially close to the peak, are very small and often cannot be measured accurately. In contrast, the velocity of each component along the line of sight is precisely known. We assume that the clouds are spherical, with similar velocity-position gradients along and perpendicular to the line of sight, allowing us to use $l$ and $\Delta V$ to represent the maximum spatial and velocity extent in any direction. We approximate each cloud as an ensemble of overlapping sub-spheres, each comprising molecules in a channel velocity interval and each giving rise to a measured maser component. We treat the features making up the multiple-peak $\mathrm{S}$ Per clouds as if they were distinct, since amplification probably only takes place along a path length similar to $l$ (not the entire depth of the cloud, since the velocity change is too great). The peak component samples the full depth, $l$, of the feature at its centre, but the depth of a sub-sphere corresponding to a component in the line wings is limited by the physical depth $l_{v}$ of a chord through the cloud at an offset position $\delta l$ determined by the local velocity gradient. We assume that $\delta l$ is proportional to the velocity offset from the feature peak, $\delta v$, for that component. This allows us to use $\delta l / 0.5 l=\delta v / 0.5 \Delta V$. By simple trigonometry, $(0.5 l)^{2}=\left(0.5 l_{v}\right)^{2}+\delta l^{2}$, leading to $l_{v} / l=\sqrt{1-\delta v^{2} /(0.5 \Delta V)^{2}}$.

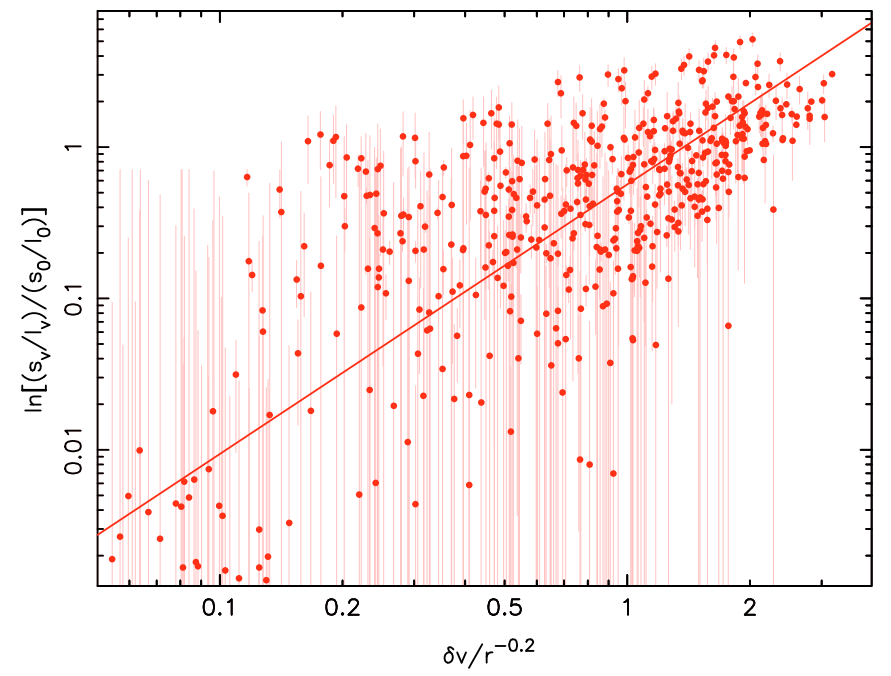

Fig. A.1. Variation of component size across the line profile for S Per in 1994. The size of maser components $s_{v}$ relative to the size of the component at the line centre $s_{0}$ as a function of offset from the line centre, weighted by the relative effective path length, as in Eq. (A.2). The line is a least-squares error-weighted fit to this relationship.

Equation (A.1) is derived from expressions for amplification-bounded masers, so it is only applicable within features where $s_{v}>s_{0}$, excluding much of the U Her and $\mathrm{U}$ Ori data from this analysis. We only consider features with Gaussian line profiles fitted with better than $3 \sigma$ accuracy $\left(N_{\text {fit }}\right.$ in Table 2), and where $\delta v / 0.5 \Delta V>1$ for both wings. The number of components meeting these criteria, $N \beta$, is given in Table 3.

We now take $l_{v}$ in Eq. (6) to represent the path length producing the amplification of a component at velocity offset $\delta v$ of size $s_{v}$ and thus replace $s_{v} / s_{0}$ with $\left(s_{v} / 0.5 l_{v} l\right) /\left(s_{0} / 0.5 l l\right)$ in Eq. (A.1). Using the more precise velocity measurements as surrogates for position measurements leads to

$$
\begin{aligned}
& \ln \left(\frac{s_{v} / l_{v}}{s_{0} / l}\right) \propto \frac{1}{2}\left(\frac{\delta v}{r^{-0.2}}\right)^{2} \\
& \text { so } \ln \left[\left(\frac{s_{v}}{s_{0}}\right) / \sqrt{1-\frac{\delta v^{2}}{(0.5 \Delta V)^{2}}}\right] \propto \frac{1}{2}\left(\frac{\delta v}{r^{-0.2}}\right)^{\beta}
\end{aligned}
$$

where $\beta=2$.

Table 3 gives values of $\beta$ obtained by error-weighted leastsquares fits to this relationship for all epochs, for features where $\Delta V_{1 / 2}>3 \sigma_{\Delta V_{1 / 2}}$. Results in italics are of low significance and suggest that this model is probably not applicable to the majority of features for these epochs.

All the values of $\beta$ are roughly $50 \%$ greater (i.e. closer to the predicted quadratic relationship) than the equivalent values of the exponent obtained by fitting to Eq. (A.1).

The $1994 \mathrm{~S}$ Per data, shown in Fig. A.1, give $\beta \approx 1.8$, closest to the expected value. The other sources with significant measurements have $\beta \approx 0.9$. Many factors could contribute to the lower values of $\beta$, such as deviations from spherical symmetry and non-linear velocity gradients. The closer angular spacing seen in many cases for brighter components compared with the displacement of the faintest from the line centre, means that $\delta v$ is not a perfect surrogate for $\delta l$. 
A. M. S. Richards et al.: Observational evidence for the shrinking of bright maser spots

\section{References}

Alcock, C., \& Ross, R. R. 1986, ApJ, 305, 837

Alvarez, R., \& Mennessier, M.-O. 1997, A\&A, 317, 761

Bains, I., Cohen, R. J., Louridas, A., et al. 2003, MNRAS, 342, 8

Cenarro, A. J., Peletier, R. F., Sánchez-Blázquez, P., et al. 2007, MNRAS, 374, 664

Chapman, J. M., \& Cohen, R. J. 1985, MNRAS, 212, 375

Chapman, J. S., Cohen, R. J., \& Saikia, D. J. 1991, MNRAS, 249, 227

Chapman, J. S., Sivagnanam, P., Cohen, R. J., \& LeSqueren, A. M. 1994, MNRAS, 268, 475

Cohen, R. J. 1987, in Circumstellar Matter, ed. I. Appenzeller, \& C. Jordan (Reidel), IAU Symp., 122, 229

Cooke, B., \& Elitzur, M. 1985, ApJ, 295, 175

de Jong, T. 1973, A\&A, 26, 297

Diamond, P. J., Johnston, K. J., Chapman, J. M., et al. 1987, A\&A, 174, 95

Elitzur, M. 1990, ApJ, 363, 628

Elitzur, M. 1992, Astronomical Masers (Kluwer)

Elitzur, M., Hollenbach, D. J., \& McKee, C. F. 1992, ApJ, 394, 221 (EHM92)

Etoka, S., \& Le Squeren, A. M. 1997, A\&A, 321, 877

Genzel, R., Downes, D., Schneps, M. H., et al. 1981, ApJ, 247, 1039

Humphreys, R. M. 1974, ApJ, 188, 75

Kirrane, T.-M. 1987, Ph.D. Thesis, University of Manchester

Lekht, E. E., Mendoza-Torres, J. E., Pashchenko, M. I., \& Berulis, I. I. 1999, A\&A, 343, 241

Lekht, E. E., Rudnitskij, G. M., Mendoza-Torres, J. E., \& Tolmachev, A. M. 2005, A\&A, 437, 127

Levesque, E. M., Massey, P., Olsen, K. A. G., et al. 2005, ApJ, 628, 973

Mayne, N. J., \& Naylor, T. 2008, MNRAS, 386, 261

Mendoza-Torres, J. E., Lekht, E. E., Pashchenko, M. I., \& Berulis, I. I. 1997, A\&AS, 126, 257
Monnier, J. D., Millan-Gabet, R., Tuthill, P. G., et al. 2004, ApJ, 605, 436

Murakawa, K., Yates, J. A., Richards, A. M. S., \& Cohen, R. J. 2003, MNRAS, 344,1

Nedoluha, G. E., \& Watson, W. D. 1992, ApJ, 384, 185

Nyman, L.-A., Johansson, L. E. B., \& Booth, R. S. 1986, A\&A, 160, 352

Olofsson, H., Lindqvist, M., Nyman, L.-A., \& Winnberg, A. 1998, A\&A, 329, 1059

Pataki, L., \& Kolena, J. 1974, Bull. Am. Astr. Soc., 6, 340

Ragland, S., Traub, W. A., Berger, J.-P., et al. 2006, ApJ, 652, 650

Reid, M. J., \& Moran, J. M. 1988, Galactic and Extragalactic Radio Astronomy (Springer-Verlag), 225

Richards, A. M. S. 1997, Ph.D. Thesis, University of Manchester

Richards, A. M. S., Yates, J. A., \& Cohen, R. J. 1996, MNRAS, 282, 665

Richards, A. M. S., Yates, J. A., \& Cohen, R. J. 1998, MNRAS, 299, 319

Richards, A. M. S., Yates, J. A., \& Cohen, R. J. 1999a, MNRAS, 306, 954 (RYC99)

Richards, A. M. S., Yates, J. A., Cohen, R. J., \& Bains, I. 1999b, in Asymptotic Giant Branch Stars, ed. T. Le Bertre, A. Lèbre, \& C. Waelkens (ASP), IAU Symp., 191, 315

Rudnitskij, G. M., Mendoza-Torres, J. E., Pashchenko, M. I., \& Berulis, I. I. 2000, A\&AS, 146, 385

Samus, N. N., Durlevich, O. V., et al. 2008, VizieR Online Data Catalog, 1, 2025

Strelnitski, V. 2007, in IAU Symp., 242, 25

van Leeuwen, F. 2007, A\&A, 474, 653

Vlemmings, W. H. T., \& van Langevelde, H. J. 2005, A\&A, 434, 1021

Vlemmings, W. H. T., \& van Langevelde, H. J. 2007, A\&A, 472, 547

Vlemmings, W. H. T., van Langevelde, H. J., \& Diamond, P. J. 2005, A\&A, 434, 1029

Yates, J. A., \& Cohen, R. J. 1994, MNRAS, 270, 958

Zubko, V., \& Elitzur, M. 2000, ApJ, 544, L137 